\title{
Longitudinal pulse shaping for the suppression of coherent synchrotron radiation-induced emittance growth
}

\author{
Chad Mitchell, ${ }^{*}$ Ji Qiang, and Paul Emma \\ Lawrence Berkeley National Laboratory, Berkeley, California 94720, USA
}

(Received 19 March 2013; published 25 June 2013)

\begin{abstract}
The damaging effect of coherent synchrotron radiation (CSR) on the emittance and energy spread of high-energy beams in accelerator light sources can significantly constrain the machine design and performance. We propose a mitigation approach in which the dynamical effect of the longitudinal component of CSR is suppressed by appropriately preparing the initial longitudinal current profile of the beam. In a chicane, a linear theory for the mechanism of CSR-induced emittance growth is used to demonstrate how this procedure can produce a beam whose core experiences suppressed transverse emittance growth. The dynamics of such a beam is illustrated for the Berlin-Zeuthen CSR benchmark chicane.
\end{abstract}

DOI: 10.1103/PhysRevSTAB.16.060703

PACS numbers: 41.60.Ap, 41.60.Cr, 29.27.Bd

\section{INTRODUCTION}

The performance of high-brightness light sources, such as X-ray free-electron lasers (FELs), requires short beams of high intensity for which the bend-induced collective interaction of coherent synchrotron radiation (CSR) can significantly impact the beam dynamics. As a bunch propagates through the bends of a magnetic bunch compressor, the tail-head interaction due to CSR induces increased beam energy spread and transverse emittance dilution that can degrade the downstream FEL performance [1-3]. In addition, the CSR interaction is known to generate a microbunching instability in which large-amplitude current modulations can grow from small-amplitude, shortwavelength variations in the initial current profile $[4,5]$.

These effects have been studied both analytically and numerically using a $1 \mathrm{D}$ rigid-bunch model of the CSR interaction [6-10]. It has been shown that the coherent radiation intensity can be suppressed through the shielding that results from the radiation interaction with the vacuum chamber walls [11]. In addition, a technique proposed in [12] has recently been demonstrated for mitigating the CSR-induced emittance growth through careful optimization of the optical lattice [13]. In this paper, we describe a complementary approach in which a 1D model is used to formulate and solve an optimization problem for the initial longitudinal beam profile needed to minimize CSRinduced emittance growth for a given lattice.

To motivate this problem, consider an electron bunch described by a longitudinal density $\lambda$, normalized such that

\section{*ChadMitchell@lbl.gov}

Published by the American Physical Society under the terms of the Creative Commons Attribution 3.0 License. Further distribution of this work must maintain attribution to the author(s) and the published article's title, journal citation, and DOI.

$$
\int_{-\infty}^{\infty} \lambda(z) d z=N
$$

where $N$ is the total number of particles in the bunch. The longitudinal steady-state 1D CSR wakefield generated by the bunch as it propagates in a bend takes the form

$$
W(z)=\int_{-\infty}^{z} \lambda\left(z^{\prime}\right) K_{\mathrm{CSR}}\left(z-z^{\prime}\right) d z^{\prime}
$$

where $K_{\mathrm{CSR}}$ is the steady-state CSR interaction kernel [6]. The resulting rms spread within the bunch in the CSRinduced energy change per unit length is given by

$$
\sigma_{W}=\sqrt{\left\langle W^{2}\right\rangle_{\|}-\langle W\rangle_{\|}^{2}}
$$

where for a given function $\phi$, we denote

$$
\langle\phi\rangle_{\|}=\frac{1}{N} \int_{-\infty}^{\infty} \phi(z) \lambda(z) d z
$$

This variation in the wakefield-induced energy change across the length of the bunch in the presence of dispersion results in a growth of the projected horizontal emittance of the bunch, given approximately in the bend by $[14,15]$

$$
\epsilon \approx \sqrt{\epsilon_{0}^{2}+\epsilon_{0} \beta\left(\theta \sigma_{E} / E\right)^{2}},
$$

where $\epsilon_{0}$ and $\epsilon$ are the initial and final unnormalized horizontal emittances, $\theta$ is the bending angle, $\beta$ is the Twiss function at the bend exit, $E$ is the beam energy, and $\sigma_{E}$ is the rms energy spread induced by CSR within the bend. (A more detailed estimate is given in Appendix C.) This motivates the following optimization problem: For a fixed bunch length $L$ and a number of particles $N$, find a density $\lambda$ such that (5) attains a minimum. A general solution to this problem can be constructed as follows. Let $L=b-a$ denote the desired bunch length, and consider a longitudinal wakefield of the form 


$$
W(z)=-W_{0}, \quad z \in[a, b],
$$

where $W_{0}>0$. Suppose that we find a longitudinal density $\lambda$ that is zero outside the finite interval $[a, b]$ and that satisfies (2) for all $z \in[a, b]$. In this case, the wakefield is uniform over the length of the bunch, and it is clear from (3) that $\sigma_{W}=0$. In this way, we eliminate the CSRinduced growth in projected emittance (5) while generating only a uniform energy loss along the bunch.

In Sec. II, we solve this problem to obtain the optimal longitudinal current profile for a bunch propagating through a single bend in the presence of steady-state CSR. In Sec. III, we construct a bunch with this longitudinal profile and examine its dynamics through a standard 4-bend magnetic chicane. In Sec. IV, a linear theory of wakefield-induced emittance growth is used to formulate a revised inverse problem that is designed to minimize the transverse emittance growth in a lattice when bend entry and exit CSR transient wakefields are included. As an example, Sec. V describes a numerical application to the benchmark chicane of the Berlin-Zeuthen CSR Workshop of 2002. Section VI concludes with a brief discussion. There are four appendices.

\section{TREATMENT OF A SINGLE BEND IN STEADY STATE}

The problem of inverting (2) to determine the density $\lambda$ that generates a given wakefield (6) involves solving a Volterra integral equation of the first kind [16,17]. We first obtain an analytical solution that is valid at high energies, when the ultrarelativistic form of the CSR interaction kernel can be used. This is followed by a discussion of the numerical solution for the more general case.

\section{A. Analytical solution in the ultrarelativistic case}

Consider a bunch with a longitudinal number density $\lambda$ propagating through a single bend of radius $R$. Under the condition that

$$
\frac{R}{\gamma^{3}} \frac{d \lambda}{d z} \ll \lambda
$$

where $\gamma$ is the relativistic factor, the steady-state longitudinal wakefield due to CSR is well described by using the ultrarelativistic form of (2) $[7,10]$ :

$$
W(z)=\int_{-\infty}^{z} \lambda\left(z^{\prime}\right) K_{\mathrm{CSR}}^{\circ}\left(z-z^{\prime}\right) d z^{\prime}
$$

where

$$
K_{\mathrm{CSR}}^{\circ}(\zeta)=\frac{2 r_{c} m c^{2}}{3^{4 / 3} R^{2 / 3} \zeta^{4 / 3}},
$$

and $r_{c}=e^{2} /\left(4 \pi \epsilon_{0} m c^{2}\right)$ denotes the classical electron radius. After integrating by parts, we can also write this in the form

$$
W(z)=-\int_{-\infty}^{z} \frac{\kappa}{\left(z-z^{\prime}\right)^{1 / 3}} \frac{d \lambda\left(z^{\prime}\right)}{d z^{\prime}} d z^{\prime}
$$

where

$$
\kappa=\frac{2 r_{c} m c^{2}}{3^{1 / 3} R^{2 / 3}} .
$$

Suppose that the longitudinal density $\lambda$ is zero outside a finite interval $[a, b]$. The lower limit in (10) then becomes $z^{\prime}=a$, and (10) takes the form of a generalized Abel integral equation for the derivative $d \lambda / d z$, with exponent $\mu=1 / 3$. (See Appendix A.) Under the assumption (6) that $W(z)=-W_{0}$, the result of Appendix A gives the unique solution:

$$
\frac{d \lambda}{d z}=\frac{\sin (\pi / 3)}{\pi \kappa} \frac{W_{0}}{(z-a)^{2 / 3}}=\frac{\sqrt{3}}{2 \pi \kappa} \frac{W_{0}}{(z-a)^{2 / 3}} .
$$

The use of integration by parts requires that the density be continuous at $z=a$, so that $\lambda(a)=0$ and we have

$$
\lambda(z)=\frac{3 \sqrt{3}}{2 \pi \kappa} W_{0}(z-a)^{1 / 3} \quad a \leq z \leq b .
$$

It follows from (13) that the density must be discontinuous at $z=b$. Applying the normalization condition (1) determines the uniform value of the wakefield:

$$
W_{0}=\frac{8}{9 \sqrt{3}} \frac{N \pi \kappa}{(b-a)^{4 / 3}},
$$

and the normalized solution for the longitudinal density becomes

$$
\lambda(z)=\frac{4}{3} N \frac{(z-a)^{1 / 3}}{(b-a)^{4 / 3}}, \quad a \leq z \leq b .
$$

For completeness, note that the rms bunch length associated with (15) is given by

$$
\sigma_{z}=\sqrt{\left\langle z^{2}\right\rangle-\langle z\rangle^{2}}=\frac{3}{7} \sqrt{\frac{2}{5}} L
$$

where $L=b-a$, and the peak current $I_{\mathrm{pk}}$ is related exactly to the bunch charge $Q_{b}$ and the length $L$ by

$$
I_{\mathrm{pk}}=\frac{4}{3} \frac{Q_{b} v}{L},
$$

where $v$ is the longitudinal velocity of the beam.

Computing the wakefield generated by the density (15) using the integral (10) gives

$$
W(z)= \begin{cases}0 & z \leq a \\ -W_{0} & a<z<b \\ -W_{0} g(z) & z>b,\end{cases}
$$

where 


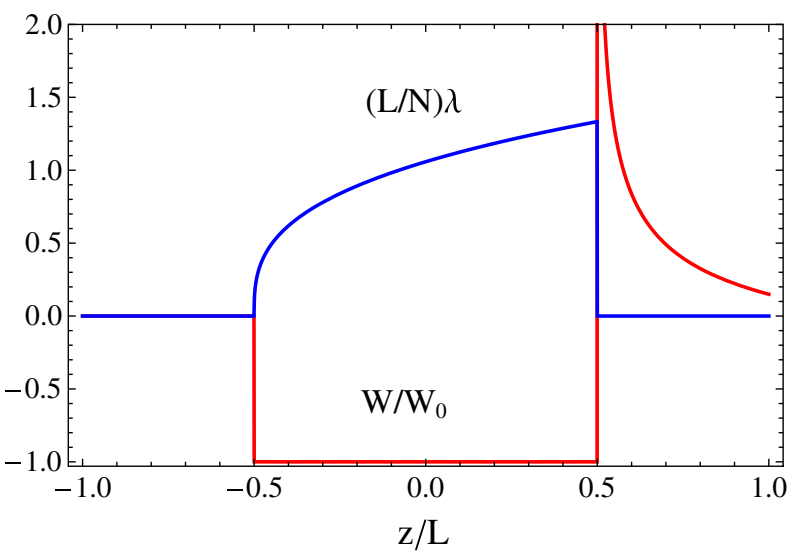

FIG. 1. (Blue) The dimensionless longitudinal density $(L / N) \lambda$, where $\lambda$ is given by (15). (Red) The dimensionless CSR wakefield $W / W_{0}$, where $W$ is given in (18) and $W_{0}$ is given in (14). Here $L$ denotes the end-to-end bunch length and $N$ denotes the total number of particles in the bunch.

$g(z)=\frac{3 \sqrt{3}}{2 \pi}\left[{ }_{2} F_{1}\left(\frac{1}{3}, \frac{1}{3} ; \frac{4}{3} ; \frac{b-a}{z-a}\right)\left(\frac{b-a}{z-a}\right)^{1 / 3}-\left(\frac{b-a}{z-b}\right)^{1 / 3}\right]$,

and ${ }_{2} F_{1}$ is the standard hypergeometric function [18]. Figure 1 illustrates the current density (15) together with its associated wakefield (18), where $a=-L / 2$ and $b=L / 2$. Note that $W$ is uniform across the length of the bunch, discontinuous at the bunch tail $(z=a)$, and singular at the bunch head $(z=b)$. We will see in the following section that when the ultrarelativistic CSR kernel $K_{\mathrm{CSR}}^{\circ}$ is replaced by the kernel $K_{\mathrm{CSR}}$ with $\gamma<\infty$, the wakefield is a continuous function that varies rapidly near the end points of the interval $[a, b]$.

Note that the derivative of $\lambda$ diverges at $z=a$, and the inequality (7) fails near this point when $\gamma<\infty$. Using (15), we expect the ultrarelativistic approximation to be accurate when

$$
z-a \gg R / 3 \gamma^{3}
$$

We therefore expect a small region near the tail of the bunch (at $z=a$ ) where the wakefield generated by (15) at $\gamma<\infty$ is not completely uniform. This case is considered in the following section.

\section{B. Numerical solution in the case $\gamma<\infty$}

When $\gamma$ is sufficiently small that (7) fails, the steadystate longitudinal wakefield due to CSR is given by (2), where the integral kernel $K_{\mathrm{CSR}}$ takes the form $[7,10]$

$$
\begin{aligned}
K_{\mathrm{CSR}}(\zeta)= & \frac{4 r_{c} m c^{2} \gamma^{4}}{R^{2}}\left\{\frac{\hat{u}^{2} / 4-1}{2\left(1+\hat{u}^{2} / 4\right)^{3}}\right. \\
& \left.+\frac{1}{\hat{u}^{2}}\left[\frac{1+3 \hat{u}^{2} / 4}{\left(1+\hat{u}^{2} / 4\right)^{3}}-\frac{1}{\left(1+\hat{u}^{2} / 12\right)^{2}}\right]\right\},
\end{aligned}
$$

with $\hat{u}$ being the root of

$$
\frac{\zeta \gamma^{3}}{R}=\frac{\hat{u}}{2}+\frac{\hat{u}^{3}}{24}
$$

In this case, the CSR wakefield integral (2) can be numerically approximated at a set of equidistant longitudinal locations $z_{k}=z_{0}+k h_{z}(k=0, \ldots, n)$ as the sum

$$
W\left(z_{k}\right)=h_{z} \sum_{k^{\prime}=0}^{k} \lambda_{k^{\prime}} w_{k-k^{\prime}}^{i g f}, \quad k=0, \ldots, n,
$$

where the values $w_{j}^{i g f}$ are determined analytically by integrating the kernel $K_{\mathrm{CSR}}$ against an appropriate set of basis functions [19]. In particular, for terms in the sum with $k^{\prime} \neq 0, k$ we use

$$
w_{j}^{i g f}=\left[I_{\mathrm{CSR}}\left(j h_{z}+h_{z} / 2\right)-I_{\mathrm{CSR}}\left(j h_{z}-h_{z} / 2\right)\right] / h_{z},
$$

where

$$
\begin{aligned}
I_{\mathrm{CSR}}(\zeta) & =-\int_{\zeta}^{\infty} K_{\mathrm{CSR}}\left(\zeta^{\prime}\right) d \zeta^{\prime} \\
& =-\frac{\gamma r_{c} m c^{2}}{R} \frac{4 \hat{u}\left(\hat{u}^{2}+8\right)}{\left(\hat{u}^{2}+4\right)\left(\hat{u}^{2}+12\right)} .
\end{aligned}
$$

The linear system (23) can be written in matrix form as

$$
\hat{W}=G \hat{\lambda},
$$

where

$$
\hat{W}_{k}=W\left(z_{k}\right), \quad \hat{\lambda}_{k}=\lambda\left(z_{k}\right) h_{z}, \quad G_{k, k^{\prime}}=w_{k-k^{\prime}}^{i g f}
$$

Note that $w_{k-k^{\prime}}^{i g f}=0$ when $k<k^{\prime}$. Thus, the matrix $G$ is lower triangular, and the system is invertible since $G_{k, k}=$ $w_{0}^{i g f} \neq 0$. Setting $\hat{W}_{k}=1$ for all $k=0, \ldots, n$, we invert the above system to obtain the values $\hat{\lambda}_{k}$, and it remains only to normalize the longitudinal density $\lambda$ according to (1). Numerical convergence issues are mentioned briefly in Appendix A.

Figure 2 illustrates the resulting numerically computed longitudinal density (red) for a bunch of total length $100 \mu \mathrm{m}$ at an energy of $100 \mathrm{MeV}$, propagating through a bend of radius $1.25 \mathrm{~m}$. This is shown together with the analytically determined ultrarelativistic density (15) in blue. Both solutions are determined under the assumption that the bunch is sufficiently far into the bend that transient CSR effects are negligible. Note that the numerically computed solution properly takes into account the short-range behavior of the CSR interaction at $100 \mathrm{MeV}$.

Figure 3 illustrates the wakefield $W / W_{0}$ along the length of the bunch as computed using (23) for the two density profiles shown in Fig. 2. The wakefield computed using the density profile (15) exhibits some variation in a small region near the tail of the bunch, while the wakefield computed using the numerically determined solution 


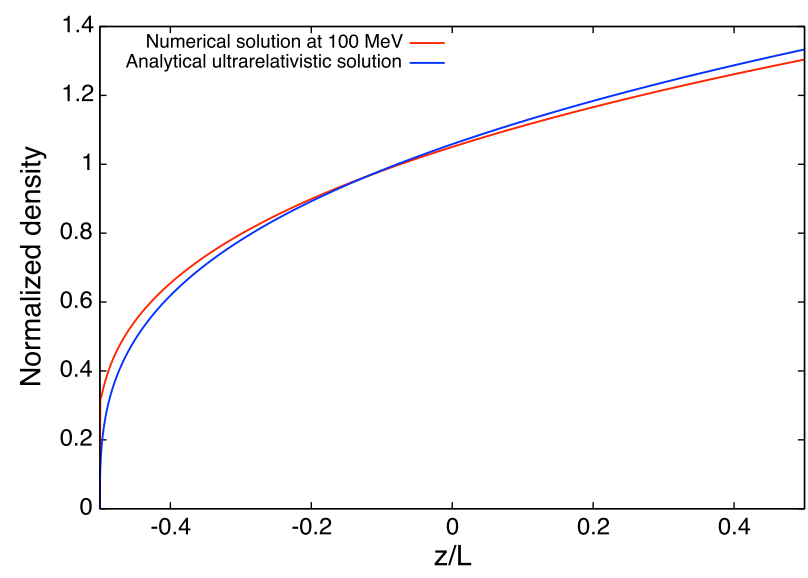

FIG. 2. (Red) Numerically computed solution of (25) for the longitudinal density. (Blue) Ultrarelativistic expression (15) for the longitudinal density. Here $L$ is the total bunch length, and the vertical axis denotes the dimensionless quantity $(L / N) \lambda$.
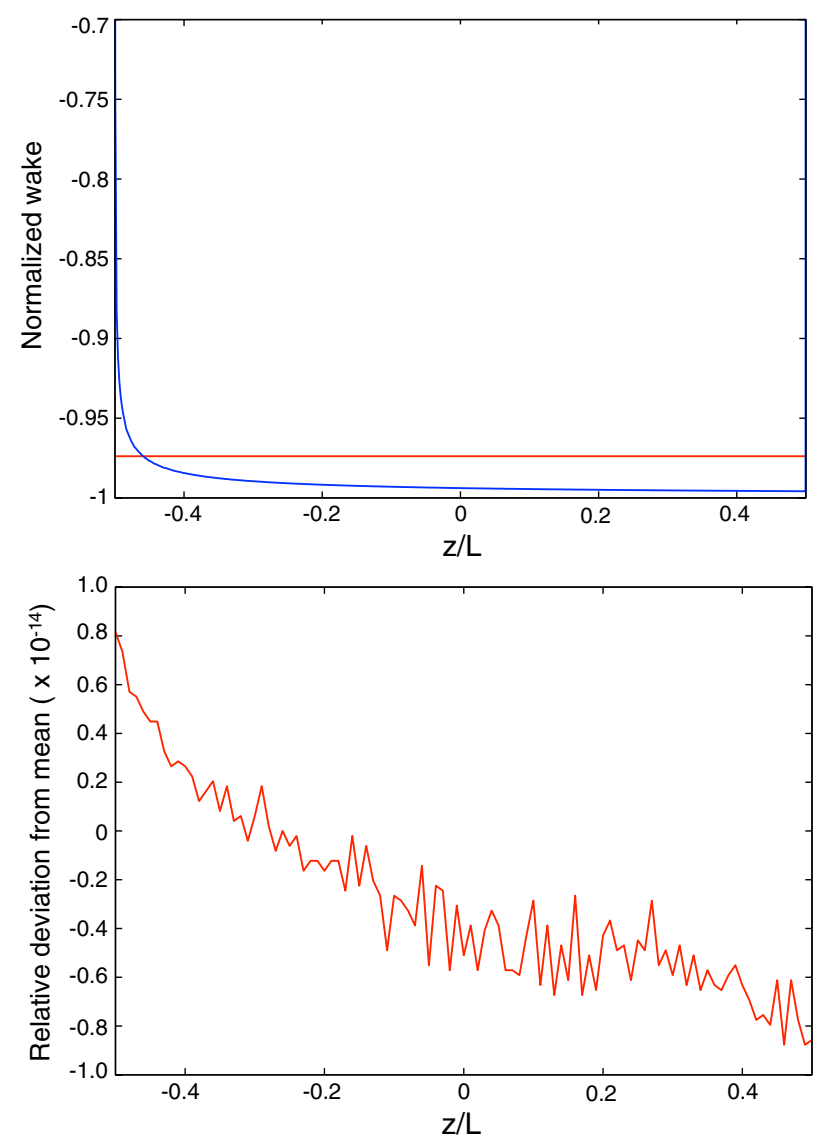

FIG. 3. The 1D CSR wakefield across the length of the bunch is shown as computed using (23) for the analytically determined density (15) (blue) and from the numerical solution of the system (25) (red). The wakefield is normalized by the quantity $W_{0}$ of (14). The lower figure illustrates the relative fluctuations in the value of the CSR wakefield as computed from the numerically determined solution. of (25) is uniform across the length of the bunch to near machine precision, as expected.

\section{DYNAMICS IN A CHICANE}

In this section, we describe how a beam with the longitudinal density (15) is affected by its longitudinal CSR wakefield as it propagates through the standard 4-bend chicane of a magnetic bunch compressor. These results indicate that under ideal linear compression, the steadystate CSR wakefield generated by (15) has no net effect on the central moments or emittance of the beam.

\section{A. Dynamics with linear optical transport}

Using the phase space variables $\mathbf{X}=\left(x, x^{\prime}, y, y^{\prime}, z, \delta\right)$, let the initial $6 \mathrm{D}$ distribution function of the beam at the entrance to the chicane take the form

$$
f_{0}(\mathbf{X})=\frac{1}{\sigma_{\delta 0} \sqrt{2 \pi}} \lambda_{0}(z) \exp \left(-\frac{(\delta-h z)^{2}}{2 \sigma_{\delta 0}^{2}}\right) f_{\perp}\left(x, x^{\prime}, y, y^{\prime}\right),
$$

where $\sigma_{\delta 0}$ is the initial uncorrelated energy spread and $h$ is the initial energy chirp. The transverse distribution $f_{\perp}$ is assumed to be Gaussian in each dimension, with

$$
f_{\perp}\left(x, x^{\prime}, y, y^{\prime}\right)=f_{x}\left(x, x^{\prime}\right) f_{y}\left(y, y^{\prime}\right) .
$$

Here $f_{x}$ is given in terms of the initial Twiss parameters $\alpha_{x 0}, \beta_{x 0}$ and the initial unnormalized emittance $\epsilon_{x 0}$ as

$$
f_{x}\left(x, x^{\prime}\right)=\frac{1}{2 \pi \epsilon_{x 0}} \exp \left[-\frac{x^{2}+\left(\beta_{x 0} x^{\prime}+\alpha_{x 0} x\right)^{2}}{2 \epsilon_{x 0} \beta_{x 0}}\right],
$$

with a similar expression for $f_{y}$. Thus, the initial longitudinal density profile of the beam is given by

$$
\lambda_{0}(z)=\int_{-\infty}^{\infty} f_{0}(\mathbf{X}) d x d x^{\prime} d y d y^{\prime} d \delta .
$$

The distribution function at a lattice location $s$ is then given through linear order in the absence of collective effects as

$$
f(\mathbf{X} ; s)=f_{0}\left[R(s)^{-1} \mathbf{X}\right],
$$

where $R(s)$ is the usual transfer matrix from the chicane entry to the location $s$. Integrating over the transverse variables gives the longitudinal density profile at $s$ :

$$
\lambda(z ; s)=\frac{C}{\sqrt{2 \pi} \mathcal{D}} \int_{-\infty}^{\infty} \lambda_{0}\left(u^{\prime}\right) \exp \left(-\frac{\left(u^{\prime}-z C\right)^{2}}{2 \mathcal{D}^{2}}\right) d u^{\prime},
$$

where

$$
C(s)=\frac{1}{1+h R_{56}(s)}
$$

is the compression factor 


$$
\mathcal{D}^{2}(s)=C^{2}\left(R_{56}^{2}(s) \sigma_{\delta 0}^{2}+\frac{\epsilon_{x 0, n} \mathcal{H}(s)}{\gamma}\right),
$$

and the parameter $\mathcal{H}$ is given by

$$
\mathcal{H}(s)=\frac{\left[\beta_{x 0} R_{51}(s)-\alpha_{x 0} R_{52}(s)\right]^{2}+R_{52}(s)^{2}}{\beta_{x 0}} .
$$

The evolution of the pulse shape is determined by the two parameters $C$ and $\mathcal{D}$. When $\mathcal{D}$ is small, the Gaussian factor appearing in the integrand of (32) becomes sharply peaked about $u^{\prime}=z C$, and in the limit $\mathcal{D} \rightarrow 0$ we find

$$
\lambda(z ; s)=C \lambda_{0}(z C) .
$$

In this limit, which we refer to as ideal compression, the effect of the chicane is to scale the longitudinal coordinate by the compression factor $C$, leaving the longitudinal bunch shape unchanged.

To study the case of nonideal compression, let $L_{f}=$ $L / C(s)$ denote the compressed bunch length at $s$. Defining scaled dimensionless quantities $\bar{z}=z / L_{f}$ and $\bar{\lambda}=$ $\left(L_{f} / N\right) \lambda$, we can write (32) as

$$
\begin{aligned}
\bar{\lambda}(\bar{z} ; s) & =\frac{1}{\sqrt{2 \pi} \hat{\mathcal{D}}} \int_{-\infty}^{\infty} \bar{\lambda}_{0}\left(\bar{z}^{\prime}\right) \exp \left(\frac{-\left(\bar{z}-\bar{z}^{\prime}\right)^{2}}{2 \hat{\mathcal{D}}^{2}}\right) d \bar{z}^{\prime} \\
& =\frac{1}{\sqrt{2 \pi} \hat{\mathcal{D}}} \frac{4}{3} \int_{-1 / 2}^{1 / 2}\left(\bar{z}^{\prime}+\frac{1}{2}\right)^{1 / 3} \exp \left(\frac{-\left(\bar{z}-\bar{z}^{\prime}\right)^{2}}{2 \hat{\mathcal{D}}^{2}}\right) d \bar{z}^{\prime} .
\end{aligned}
$$

The shape of the compressed pulse is controlled by the dimensionless parameter $\hat{\mathcal{D}}=\mathcal{D} / L$, given explicitly by

$$
\hat{\mathcal{D}}^{2}=\frac{R_{56}^{2} \sigma_{\delta 0}^{2}+\epsilon_{x 0, n} \mathcal{H} / \gamma}{L_{f}^{2}},
$$

and the result is shown in Fig. 4 for several values of the parameter $\hat{\mathcal{D}}$. As $\hat{\mathcal{D}}$ increases, smoothing of the longitudinal density profile is visible on the scale $\Delta z / L_{f} \sim \hat{\mathcal{D}}$. For the example considered in Sec. V, the parameter $\hat{\mathcal{D}}$ ranges from 0\%-6\%. (See Fig. 7.)

As the longitudinal density of the bunch evolves in the lattice, the longitudinal CSR wakefield will also evolve. Let $W_{f}=W_{0} C(s)^{4 / 3}$, and define the dimensionless quantity $\bar{W}=W / W_{f}$. Using arguments similar to those above, we find

$$
\bar{W}(\bar{z} ; s)=\frac{1}{\sqrt{2 \pi} \hat{\mathcal{D}}} \int_{-\infty}^{\infty} \bar{W}^{(0)}\left(\bar{z}^{\prime}\right) \exp \left(\frac{-\left(\bar{z}-\bar{z}^{\prime}\right)^{2}}{2 \hat{\mathcal{D}}^{2}}\right) d \bar{z}^{\prime},
$$

where $\bar{z}=z / L_{f}, \bar{W}^{(0)}=W^{(0)} / W_{0}$, and $W^{(0)}$ is the initial wakefield given by (18). Figure 5 illustrates this result as a function of $\bar{z}$ for several values of the parameter $\hat{\mathcal{D}}$. Notice that due to the longitudinal spreading induced by the initial energy spread and transverse emittance of the beam,

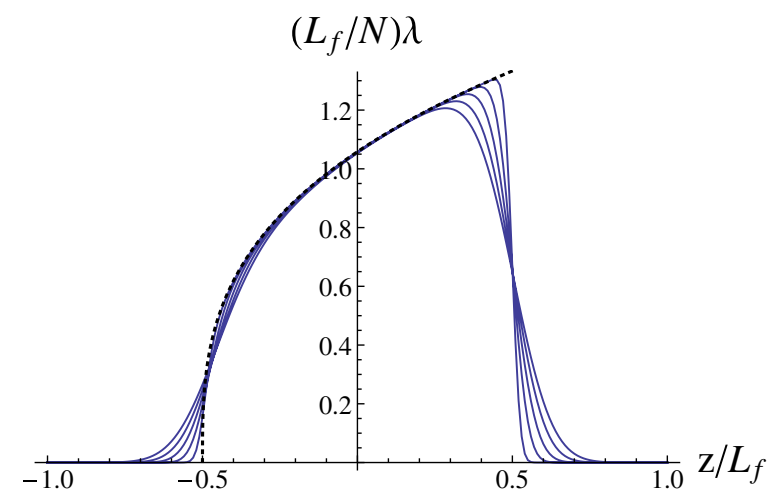

FIG. 4. The evolution of the longitudinal density $\lambda$ within a chicane in the absence of collective effects is shown for the values $\hat{\mathcal{D}}=2 \%, 4 \%, 6 \%, 8 \%$, and $10 \%$, demonstrating the effect of nonzero initial energy spread $\sigma_{\delta 0}$ and transverse emittance $\epsilon_{x 0, n}$ on the bunch profile. The dashed line illustrates the initial density and $L_{f}=L / C$ denotes the compressed bunch length.

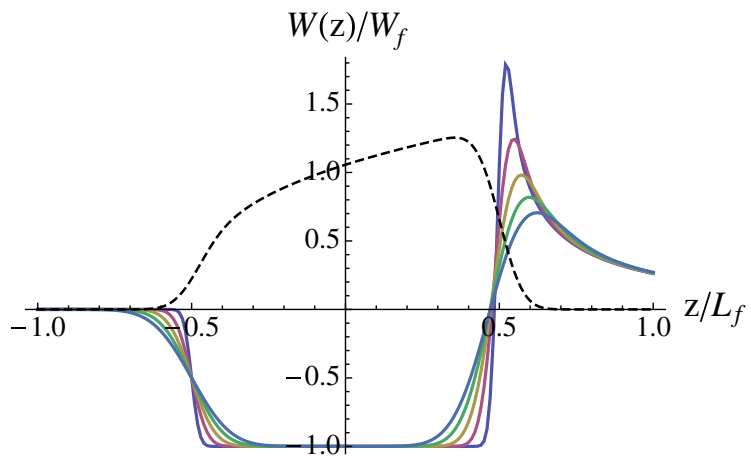

FIG. 5. (Solid) The 1D CSR wakefield that would be generated by the bunches of Fig. 4, shown for the values $\hat{\mathcal{D}}=2 \%, 4 \%, 6 \%$, $8 \%$, and $10 \%$, demonstrating the effect of nonzero initial energy spread $\sigma_{\delta 0}$ and transverse emittance $\epsilon_{x 0, n}$ on the longitudinal CSR wakefield. (Dashed) The current profile $\left(L_{f} / N\right) \lambda$ for the case $\hat{\mathcal{D}}=6 \%$ is shown for comparison, illustrating that particles near the head of the bunch at $z / L_{f}=1 / 2$ will experience a CSR-induced energy kick.

particles within a distance $\Delta z$ of the bunch head can slip forward into a region of large accelerating wakefield. Likewise, particles within the same distance of the bunch tail can slip backward into a region where the longitudinal wakefield varies from its uniform value along the bunch core. In Sec. V, we will see that the bunch experiences a growth in horizontal projected emittance that is due entirely to particles in these two regions.

\section{B. Dynamics with nonlinear optical transport}

The presence of nonlinear beam optics can lead to a distortion of the initial longitudinal current profile (15) downstream within the bunch compressor. As a result, the CSR wakefield generated in the bends may be 
nonuniform, introducing energy spread and transverse emittance growth. In this section we review results which show that, by modifying the initial longitudinal energy profile appropriately, nonlinear effects on the dynamics of the current profile can be eliminated through third order [20].

We will consider only the nonlinear dynamics in the longitudinal phase space. Assume that the initial mean energy of a longitudinal slice $z_{i}$ at the chicane entry is given by

$$
\delta\left(z_{i}\right)=\alpha_{1} z_{i}+\alpha_{2} z_{i}^{2}+\alpha_{3} z_{i}^{3}+O\left(z_{i}^{4}\right),
$$

where $\alpha_{1}$ is the linear energy-bunch length correlation that is required for bunch compression. The nonlinear transfer map through third order in the chicane is given by

$z_{f}=z_{i}+R_{56} \delta_{i}+T_{566} \delta_{i}^{2}+U_{566} \delta_{i}^{3}+O\left(\delta_{i}^{4}\right), \quad \delta_{f}=\delta_{i}$.

Using (40) gives

$$
\begin{aligned}
z_{f}= & \left(1+\alpha_{1} R_{56}\right) z_{i}+\left(\alpha_{1}^{2} T_{566}+\alpha_{2} R_{56}\right) z_{i}^{2} \\
& +\left(\alpha_{1}^{3} U_{5666}+2 T_{566} \alpha_{1} \alpha_{2}+\alpha_{3} R_{56}\right) z_{i}^{3}+O\left(z_{i}\right)^{4} .
\end{aligned}
$$

Nonlinear terms in (42) can be eliminated through third order by choosing

$$
\alpha_{2}=-\alpha_{1}^{2} \frac{T_{566}}{R_{56}}, \quad \alpha_{3}=\frac{1}{R_{56}}\left(-\alpha_{1}^{3} U_{5666}+2 \alpha_{1}^{3} \frac{T_{566}^{2}}{R_{56}}\right) .
$$

For a chicane [15],

$$
T_{566} \approx-\frac{3}{2} R_{56}, \quad U_{5666} \approx 2 R_{56} .
$$

Using these results in (43) gives

$$
\delta\left(z_{i}\right)=\bar{z}_{i}+\frac{3}{2} \bar{z}_{i}^{2}+\frac{5}{2} \bar{z}_{i}^{3}+O\left(\bar{z}_{i}^{4}\right),
$$

where

$$
\bar{z}_{i}=\alpha_{1} z_{i} .
$$

By introducing nonlinear terms into the initial energy profile according to (45), the expression (42) becomes $z_{f}=z_{i} / C$. As a result, the longitudinal bunch shape in the presence of nonlinear effects through third order will evolve as described in Sec. III A. (See Fig. 9.)

\section{Dynamics in the presence of a uniform CSR wakefield}

In Sec. III A, we saw that the linear evolution of a bunch with the initial distribution function (27) through a chicane in the absence of collective effects is well described by a simple scaling of the longitudinal coordinate $z$ (ideal compression) when the parameter $\hat{\mathcal{D}}$ is sufficiently small. In the presence of nonlinear optical transport, this behavior can be restored by shaping the initial energy profile of the bunch as described in Sec. III B. Under these conditions, we have for a beam with the initial longitudinal density (15):

$$
\lambda(z ; s)=C \lambda_{0}(z C)=\frac{4}{3} N \frac{(z-\bar{a})^{1 / 3}}{(\bar{b}-\bar{a})^{4 / 3}}
$$

where $\bar{a}=a / C$ and $\bar{b}=b / C$. Following the results of Sec. II, it follows that at each location $s$ in the lattice the longitudinal wakefield due to CSR is uniform across the length of the bunch, with a value given according to

$$
W(s)=-W_{0} C(s)^{4 / 3},
$$

where $W_{0}$ is given in (14). We can relate the evolution of the distribution function in the presence of CSR (denoted $f_{\text {CSR }}$ ) and in the absence of CSR (denoted $f$ ) in this case as follows.

Define the 6-tuple

$$
\Gamma(s)=(0,0,0,0,0, W(s) / E),
$$

where $E$ is the beam energy. To linear order, the phase space coordinate of each particle in the presence of CSR satisfies a differential equation of the form

$$
\mathbf{X}^{\prime}(s)=A(s) \mathbf{X}(s)+\Gamma(s), \quad \mathbf{X}(0)=\mathbf{X}_{0},
$$

where the matrix $A$ is determined by the externally applied fields. The exact solution is given by variation of parameters [21] as

$$
\mathbf{X}(s)=R(s) \mathbf{X}_{0}+\int_{0}^{s} R(s) R^{-1}(\tau) \boldsymbol{\Gamma}(\tau) d \tau,
$$

where $R$ is the linear transfer matrix, which satisfies

$$
R^{\prime}(s)=A(s) R(s), \quad R(0)=I,
$$

and $I$ is the $6 \times 6$ identity matrix. Solving for $\mathbf{X}_{0}$ in terms of $\mathbf{X}(s)$ gives

$$
\mathbf{X}_{0}=R^{-1}(s) \mathbf{X}(s)-\int_{0}^{s} R^{-1}(\tau) \boldsymbol{\Gamma}(\tau) d s .
$$

The distribution function therefore evolves as

$$
f_{\mathrm{CSR}}(\mathbf{X} ; s)=f_{0}\left(R^{-1}(s) \mathbf{X}-\int_{0}^{s} R^{-1}(\tau) \mathbf{\Gamma}(\tau) d \tau\right) .
$$

Comparing this with the distribution function in the absence of CSR (31) we find that

$$
f_{\mathrm{CSR}}(\mathbf{X} ; s)=f[\mathbf{X}-\Delta \mathbf{X}(s) ; s],
$$

where

$$
\Delta \mathbf{X}(s)=\int_{0}^{s} R(\tau \rightarrow s) \boldsymbol{\Gamma}(\tau) d \tau
$$

In components, (56) gives 


$$
\begin{aligned}
\Delta x(s) & =\frac{1}{E} \int_{0}^{s} R_{16}(\tau \rightarrow s) W(\tau) d \tau, \\
\Delta x^{\prime}(s) & =\frac{1}{E} \int_{0}^{s} R_{26}(\tau \rightarrow s) W(\tau) d \tau, \\
\Delta z(s) & =\frac{1}{E} \int_{0}^{s} R_{56}(\tau \rightarrow s) W(\tau) d \tau, \\
\Delta \delta(s) & =\frac{1}{E} \int_{0}^{s} W(\tau) d \tau,
\end{aligned}
$$

where the quantity $W$ is given by (48).

The effect of the uniform CSR wakefield generated by the density (15) under ideal compression is therefore to introduce a translation of the beam distribution function in phase space given by (57). As a result, the influence of CSR affects the location of the beam centroid, but has no net effect on the central beam moments or emittances.

\section{Dynamics in the presence of a nonuniform CSR wakefield}

The discussion of the previous section demonstrates that a bunch with the initial longitudinal density profile (15) will maintain this profile under ideal compression in a chicane, resulting in no net projected emittance growth in the presence of steady-state CSR. In a realistic system, however, transient CSR wakefields [7] occur near the transitions between bends and drifts, resulting in a CSR interaction that varies according to the bunch location within the lattice. Such a wakefield takes the general form:

$$
W(z, s)=\int_{-\infty}^{z} \lambda(z ; s) K_{\mathrm{CSR}}\left(z+s, z^{\prime}+s\right) d z^{\prime} .
$$

A longitudinal density profile which generates a uniform wakefield at one location in the lattice will not, in general, generate a uniform wakefield elsewhere due to the $s$ dependence of the kernel $K_{\mathrm{CSR}}$.

To linear order in the externally applied fields, the phase space coordinate of a particle in the presence of such a longitudinal wakefield satisfies a differential equation with a form similar to (50):

$$
\mathbf{X}^{\prime}(s)=A(s) \mathbf{X}(s)+\boldsymbol{\Gamma}^{[\lambda]}[\mathbf{X}(s), s], \quad \mathbf{X}(0)=\mathbf{X}_{0},
$$

where the inhomogeneous term describes the change in particle energy due to the longitudinal wakefield:

$$
\boldsymbol{\Gamma}^{[\lambda]}[\mathbf{X}(s), s]=\{0,0,0,0,0, W[z(s), s] / E\},
$$

and the notation $\boldsymbol{\Gamma}^{[\lambda]}$ denotes that $W$ in (60) is determined self-consistently from the longitudinal density profile of the beam according to (58). The system (59) can be rewritten as the integral equation:

$$
\mathbf{X}(s)=R(s) \mathbf{X}_{0}+\int_{0}^{s} R(\tau \rightarrow s) \Gamma^{[\lambda]}[\mathbf{X}(\tau), \tau] d \tau .
$$

Now (61) can be solved using an iterative procedure as follows. Let

$$
\mathbf{X}^{(0)}(s)=R(s) \mathbf{X}_{0}
$$

denote the zero-order solution, which describes the dynamics in the absence of the wakefield $W$. Using this solution in the integral appearing on the right-hand side of (61) gives the first-order solution,

$$
\mathbf{X}^{(1)}(s)=\mathbf{X}^{(0)}(s)+\int_{0}^{s} R(\tau \rightarrow s) \boldsymbol{\Gamma}^{[\lambda]}\left[\mathbf{X}^{(0)}(\tau), \tau\right] d \tau,
$$

and the solution of order $n \geq 1$ is given by

$$
\mathbf{X}^{(n)}(s)=\mathbf{X}^{(0)}(s)+\int_{0}^{s} R(\tau \rightarrow s) \Gamma^{[\lambda]}\left[\mathbf{X}^{(n-1)}(\tau), \tau\right] d \tau .
$$

Consider the evolution of particles within a single longitudinal slice of the bunch, defined by the initial coordinate $z=u$. To make use of the expression (63), we will make the additional approximation that the unperturbed dynamics is described by ideal compression, so that the final longitudinal location of the slice is given by

$$
z(s)=u / C(s) .
$$

Particles within a longitudinal slice with the initial location $u$ experience a net offset as a result of the CSR wakefield given according to (63) at a lattice location $s$ by

$$
\begin{aligned}
& \Delta x(u ; s)=\frac{1}{E} \int_{0}^{s} R_{16}(\tau \rightarrow s) W[u / C(\tau), \tau] d \tau, \\
& \Delta x^{\prime}(u ; s)=\frac{1}{E} \int_{0}^{s} R_{26}(\tau \rightarrow s) W[u / C(\tau), \tau] d \tau, \\
& \Delta z(u ; s)=\frac{1}{E} \int_{0}^{s} R_{56}(\tau \rightarrow s) W[u / C(\tau), \tau] d \tau, \\
& \Delta \delta(u ; s)=\frac{1}{E} \int_{0}^{s} W[u / C(\tau), \tau] d \tau,
\end{aligned}
$$

where the quantity $W$ is determined by (58). In the following section, we will see that the variation of the quantities $\Delta x$ and $\Delta x^{\prime}$ with the slice coordinate $u$ is directly related to the CSR-induced projected emittance growth.

\section{SUPPRESSION OF CSR-INDUCED EMITTANCE GROWTH}

In Sec. II, an integral equation was solved to determine the longitudinal density profile that will produce uniform CSR-induced energy loss along the bunch length in the presence of steady-state CSR in a bend. In this section, the treatment is generalized to include the effects of transient CSR throughout a chicane, resulting in an initial longitudinal density profile that will produce suppressed CSR-induced emittance growth at the chicane exit.

Treating the beam as a collection of longitudinal slices whose centroids evolve according to (66) under the influence of CSR, the CSR-induced offset in the transverse phase space of a given beam slice at the exit of the system (denoted by $s=s_{f}$ ) can be written in terms of the initial longitudinal density $\lambda_{0}$ in the form 


$$
\begin{aligned}
\Delta x(u) & =\frac{1}{E} \int_{-\infty}^{u} \lambda_{0}\left(u^{\prime}\right) K_{\mathrm{CSR}}^{\mathrm{int}, x}\left(u, u^{\prime}\right) d u^{\prime}, \\
\Delta x^{\prime}(u) & =\frac{1}{E} \int_{-\infty}^{u} \lambda_{0}\left(u^{\prime}\right) K_{\mathrm{CSR}}^{\mathrm{int}, x^{\prime}}\left(u, u^{\prime}\right) d u^{\prime},
\end{aligned}
$$

where the modified kernels,

$$
\begin{aligned}
K_{\mathrm{CSR}}^{\mathrm{int}, x}\left(u, u^{\prime}\right)= & \int_{0}^{s_{f}} R_{16}\left(\tau \rightarrow s_{f}\right) K_{\mathrm{CSR}}[u / C(\tau) \\
& \left.+\tau, u^{\prime} / C(\tau)+\tau\right] d \tau, \\
K_{\mathrm{CSR}}^{\mathrm{int}, x^{\prime}}\left(u, u^{\prime}\right)= & \int_{0}^{s_{f}} R_{26}\left(\tau \rightarrow s_{f}\right) K_{\mathrm{CSR}}[u / C(\tau) \\
& \left.+\tau, u^{\prime} / C(\tau)+\tau\right] d \tau,
\end{aligned}
$$

represent the cumulative transverse effect of the longitudinal CSR interaction throughout the chicane.

The horizontal projected emittance at the exit of the chicane is given in terms of the centroid offsets (67) and the final slice Twiss functions $(\alpha, \beta, \gamma)$ and slice horizontal emittance $\epsilon_{0}$ by (Appendix B)

$$
\epsilon^{2}=\epsilon_{0}^{2}+\epsilon_{0}\left(\beta \sigma_{x^{\prime} \|}^{2}+\gamma \sigma_{x \|}^{2}+2 \alpha\left\langle X X^{\prime}\right\rangle_{\|}\right)+\epsilon_{\|}^{2},
$$

where

$$
\begin{aligned}
\sigma_{x \|}^{2} & =\left\langle\Delta x^{2}\right\rangle_{\|}-\langle\Delta x\rangle_{\|}^{2}, \quad \sigma_{x^{\prime} \|}^{2}=\left\langle\Delta x^{\prime 2}\right\rangle_{\|}-\left\langle\Delta x^{\prime}\right\rangle_{\|}^{2}, \\
\left\langle X X^{\prime}\right\rangle_{\|} & =\left\langle\Delta x \Delta x^{\prime}\right\rangle_{\|}-\langle\Delta x\rangle_{\|}\left\langle\Delta x^{\prime}\right\rangle_{\|},
\end{aligned}
$$

and

$$
\epsilon_{\|}^{2}=\sigma_{x \|}^{2} \sigma_{x^{\prime} \|}^{2}-\left\langle X X^{\prime}\right\rangle_{\|}^{2}
$$

Here the average $\langle\cdot\rangle_{\|}$is taken across slices as in (4).

It is not difficult to show that $\epsilon \geq \epsilon_{0}$, and this absolute minimum is attained only when all slices in the beam are aligned so that

$$
\Delta x(u)=\langle\Delta x\rangle_{\|}, \quad \Delta x^{\prime}(u)=\left\langle\Delta x^{\prime}\right\rangle_{\|},
$$

independently of the slice coordinate $u$. To eliminate the wakefield-induced projected emittance growth, one must find an initial longitudinal density $\lambda_{0}$ (for fixed particle number $N$ and bunch length $L$ ) satisfying (70), with the centroid offsets $\Delta x$ and $\Delta x^{\prime}$ given according to (67). This results in a pair of Volterra integral equations for the density $\lambda_{0}$, which cannot in general be inverted simultaneously. Instead, rigorous minimization of the emittance growth requires a nonlinear optimization of (69) over the space of initial density profiles.

However, suppose that

$$
\Delta x(u)=\langle\Delta x\rangle_{\|},
$$

so that all slices are aligned in the $x-z$ phase space projection. Then it follows that $\sigma_{x \|}=\left\langle X X^{\prime}\right\rangle_{\|}=0$ and

$$
\epsilon^{2}=\epsilon_{0}^{2}+\epsilon_{0} \beta \sigma_{x^{\prime} \|}^{2}
$$

Alternatively, if we suppose that

$$
\Delta x^{\prime}(u)=\left\langle\Delta x^{\prime}\right\rangle_{\|}
$$

so that all slices are aligned in the $x^{\prime}-z$ phase space projection, then it follows that $\sigma_{x^{\prime} \|}=\left\langle X X^{\prime}\right\rangle_{\|}=0$ and

$$
\epsilon^{2}=\epsilon_{0}^{2}+\epsilon_{0} \gamma \sigma_{x \|}^{2}
$$

In this way, we see how distortions in the $x-z$ and $x^{\prime}-z$ planes each relatively contribute to the final projected emittance.

By comparing (72) and (74), we see that it is sufficient to invert one of the linear problems (71) or (73) when the resulting contribution from $\sigma_{x \| \mid}$ or $\sigma_{x^{\prime} \|}$ to the emittance is sufficiently small. For the parameters considered in Sec. V, for example, we have that

$\left.\left.\frac{\sigma_{x^{\prime} \|}^{2}}{\gamma_{x}}\right|_{\Delta x=\langle\Delta x\rangle_{\|}} \approx 16 \epsilon_{0} \gg 0.01 \epsilon_{0} \approx \frac{\sigma_{x \|}^{2}}{\beta_{x}}\right|_{\Delta x^{\prime}=\left\langle\Delta x^{\prime}\right\rangle_{\|}}$.

Thus, we wish to search for an initial density $\lambda_{0}$ on the interval $[a, b]$ such that $\Delta x^{\prime}(u)$ is independent of $u$, as in (73). Using (67b), this gives the integral equation:

$$
\Lambda=\int_{-\infty}^{u} \lambda_{0}\left(u^{\prime}\right) K_{\mathrm{CSR}}^{\mathrm{int}, x^{\prime}}\left(u, u^{\prime}\right) d u^{\prime}, \quad u \in[a, b],
$$

for some constant $\Lambda \neq 0$.

To solve (76) numerically, it is convenient to represent the integral in terms of an integrated Green function [19], using a procedure similar to that of Sec. II B. For this purpose, we define

$$
I_{\mathrm{CSR}}^{\mathrm{int}, x^{\prime}}\left(u, u^{\prime}\right)=-\int_{-\infty}^{u^{\prime}} K_{\mathrm{CSR}}^{\mathrm{int}, x^{\prime}}\left(u, u^{\prime \prime}\right) d u^{\prime \prime} .
$$

Using (68b), (77) becomes

$$
\begin{aligned}
I_{\mathrm{CSR}}^{\mathrm{int}, x^{\prime}}\left(u, u^{\prime}\right)= & \int_{0}^{s_{f}} C(\tau) R_{26}\left(\tau \rightarrow s_{f}\right) \\
& \times I_{\mathrm{CSR}}\left[u / C(\tau)+\tau, u^{\prime} / C(\tau)+\tau\right] d \tau,
\end{aligned}
$$

where

$$
I_{\mathrm{CSR}}\left(\tau, \tau^{\prime}\right)=-\int_{-\infty}^{\tau^{\prime}} K_{\mathrm{CSR}}\left(\tau, \tau^{\prime \prime}\right) d \tau^{\prime \prime}
$$

Let us sample the lattice at equally spaced locations $\tau_{j}=\tau_{0}+j \Delta \tau(j=0, \ldots, N)$, and the bunch coordinate at equally spaced locations $u_{k}=u_{0}+k h_{z}(k=0, \ldots, n)$. Using an extended trapezoidal rule to evaluate the integral in $\tau$, we can approximate the equation (76) as

$$
\Lambda=h_{z} \sum_{k^{\prime}=0}^{k} \lambda_{0}\left(u_{k^{\prime}}\right) w_{k, k^{\prime}}^{i n t}, \quad(k=0, \ldots, n),
$$

where

$$
w_{k, k^{\prime}}^{\mathrm{int}}=\frac{\Delta \tau}{2} w_{k, k^{\prime}, 0}^{i g f}+\frac{\Delta \tau}{2} w_{k, k^{\prime}, N}^{i g f}+\Delta \tau \sum_{j=1}^{N-1} w_{k, k^{\prime}, j^{\prime}}^{i g f}
$$

and 


$$
\begin{aligned}
& w_{k, k^{\prime}, j}^{i g f} \\
& =C_{j} R_{26}\left(\tau_{j} \rightarrow s_{f}\right)\left\{I_{\mathrm{CSR}}\left(u_{k} / C_{j}+\tau_{j},\left[u_{k^{\prime}}-h_{z} / 2\right] / C_{j}+\tau_{j}\right)\right. \\
& \left.\quad-I_{\mathrm{CSR}}\left(u_{k} / C_{j}+\tau_{j},\left[u_{k^{\prime}}+h_{z} / 2\right] / C_{j}+\tau_{j}\right)\right\} / h_{z},
\end{aligned}
$$

with $C_{j}=C\left(\tau_{j}\right)$.

This gives a lower-diagonal linear system of the form

$$
\hat{\Lambda}=G^{\text {int }} \hat{\lambda}, \quad G_{k, k^{\prime}}^{\text {int }}=w_{k, k^{\prime}}^{\text {int }}
$$

where the arrays $\hat{\Lambda}$ and $\hat{\lambda}$ have components $\hat{\Lambda}_{k}=\Lambda$ and $\hat{\lambda}_{k}=\lambda_{0}\left(u_{k}\right) h_{z}$. When $G_{k, k}^{\text {int }} \neq 0$ for $k=0, \ldots, n$, we may invert this system numerically to obtain the desired density $\hat{\lambda}$. Note that the solution depends on the lattice design through the matrix elements $R_{26}$ and $R_{56}$.

\section{APPLICATION TO A BENCHMARK CHICANE}

As a numerical example, we consider a benchmark chicane first proposed in 2002 at the Berlin MiniWorkshop on CSR [22]. Figure 6 illustrates the chicane geometry, and Table I contains the lattice and beam parameters. The compression factor $C$ and the dimensionless factor $\hat{\mathcal{D}}$, which together control the longitudinal bunch shape, are shown in Fig. 7 as functions of path length $s$ through the lattice.

We first demonstrate the evolution of a bunch through the chicane in the presence of linear magnetic optics and steady-state ultrarelativistic CSR, as described in Sec. III. A bunch with the initial distribution function (27) for the parameters given in Table I was generated using 10 million particles, and its evolution in the chicane of Fig. 6 was simulated using IMPACT-Z [23] in the presence of steadystate, ultrarelativistic CSR using the wakefield model (10) with 8192 longitudinal bins. A Gaussian bunch with the same rms bunch length and Twiss parameters was tracked for comparison. Figure 8 illustrates the net CSR-induced change in the mean slice energy along the length of the bunch at three different lattice locations, indicating that the cumulative energy loss remains uniform along the length of the optimized bunch as the bunch propagates through the chicane. Note that the value of this energy loss is similar to the peak energy loss experienced for a

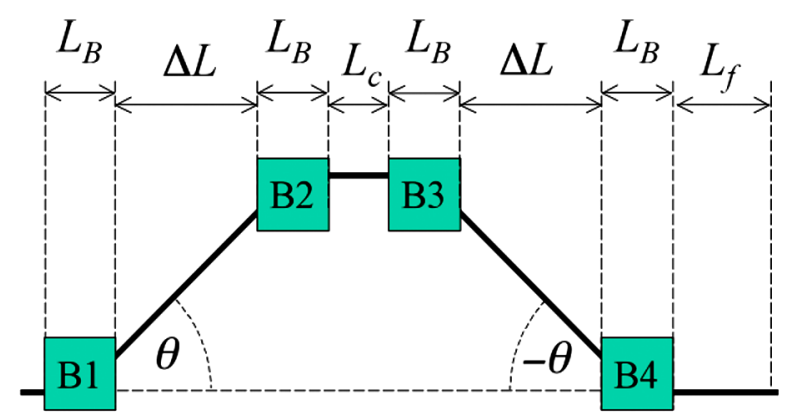

FIG. 6. Schematic of the Berlin-Zeuthen chicane lattice.
TABLE I. Parameters used for simulation of the BerlinZeuthen benchmark chicane [22].

\begin{tabular}{lccc}
\hline \hline Chicane parameters & Symbol & Value & Unit \\
\hline Bend magnet length & $L_{B}$ & 0.5 & $\mathrm{~m}$ \\
Drift length $\mathrm{B} 1 \rightarrow \mathrm{B} 2, \mathrm{~B} 3 \rightarrow \mathrm{B} 4)$ & $\Delta L$ & 5 & $\mathrm{~m}$ \\
Drift length $\mathrm{B} 2 \rightarrow \mathrm{B} 3)$ & $L_{c}$ & 1.0 & $\mathrm{~m}$ \\
Drift length (final drift) & $L_{f}$ & 2.0 & $\mathrm{~m}$ \\
Bend angle & $|\theta|$ & 2.770 & $\mathrm{deg}$ \\
Bend radius & $|R|$ & 10.35 & $\mathrm{~m}$ \\
Momentum compaction factor & $R_{56}$ & -25.0 & $\mathrm{~mm}$ \\
\hline Electron beam parameters & Symbol & Value & Unit \\
\hline Nominal energy & $E_{0}$ & 5.0 & $\mathrm{GeV}$ \\
Bunch charge & $q$ & 1.0 & $\mathrm{nC}$ \\
Uncorrelated initial rms energy spread & $\sigma_{\delta 0}$ & 2.0 & $10^{-6}$ \\
Linear energy chirp & $h$ & 36.0 & $\mathrm{~m}$ \\
Initial rms bunch length & $\sigma_{z 0}$ & 200 & $\mu \mathrm{m}$ \\
Final rms bunch length & $\sigma_{z f}$ & 20 & $\mu \mathrm{m}$ \\
Initial rms normalized emittances & $\gamma \epsilon_{x, y}$ & $1.0,1.0$ & $\mu \mathrm{m}$ \\
Initial beta functions & $\beta_{x 0, y 0}$ & 40,13 & $\mathrm{~m}$ \\
Initial alpha functions & $\alpha_{x 0, y 0}$ & 2.6 & \\
\hline \hline
\end{tabular}

Gaussian bunch, and the largest energy loss occurs in Bend 4. Figure 9 illustrates the current pulse at the entrance of this bend, indicating that the pulse shape remains nearly optimal at this location, as expected from the small value of the parameter $\hat{\mathcal{D}} \approx 0.06$. (See Fig. 4.) The relative growth
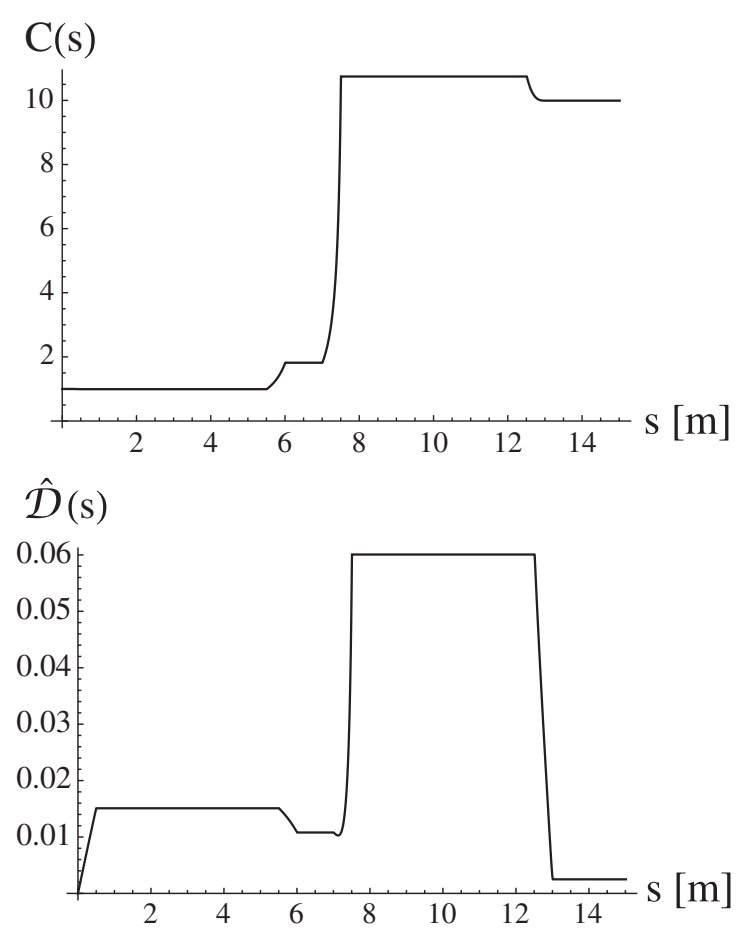

FIG. 7. (Upper) The compression factor $C$ as a function of path length through the lattice shown in Fig. 6. (Lower) The factor $\hat{\mathcal{D}}$ of (38) as a function of path length through the lattice shown in Fig. 6. 

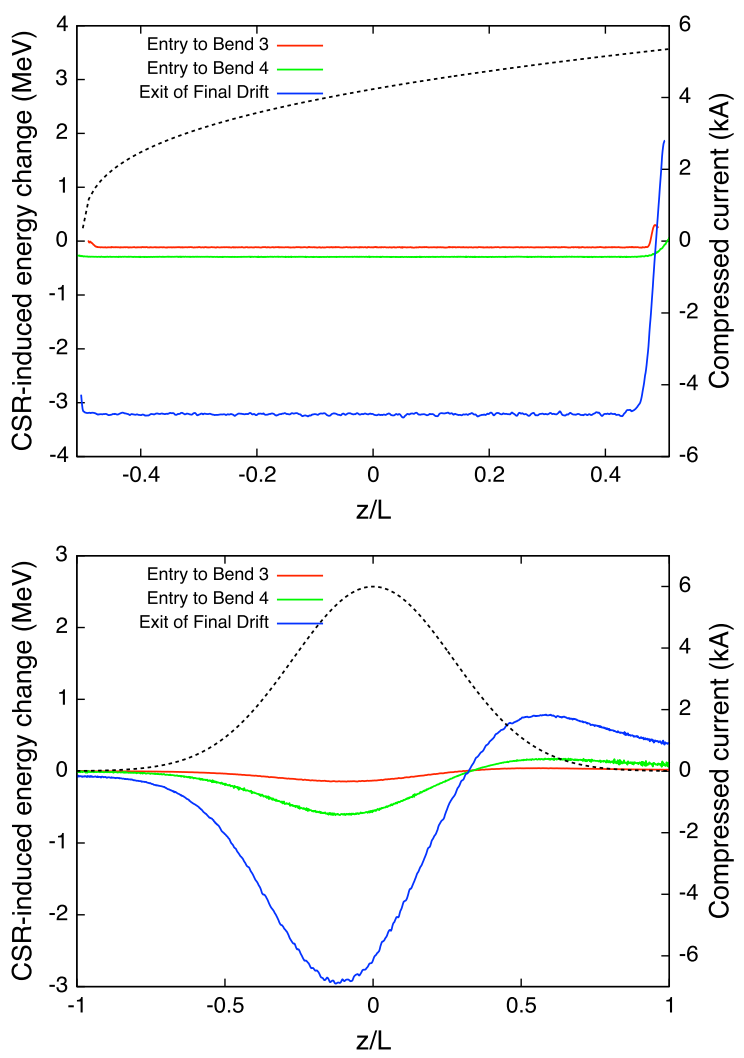

FIG. 8. (Top) The difference in mean slice energy in the presence and absence of CSR for the density given in (15). The result is shown at the entry to Bend 3 (red), the entry to Bend 4 (green), and the exit of the system (blue). The final compressed current profile is shown for reference (dashed). (Bottom) The difference in mean slice energy in the presence and absence of CSR for a Gaussian density with the same second moments.

in the transverse rms projected emittance at the exit of the system is $5 \%$ for the optimized beam profile and $19.5 \%$ for the Gaussian beam profile, while the slice emittance is unchanged in both cases. However, for the optimized beam, the projected emittance growth is confined to particles located within $7 \mu \mathrm{m}$ of the head of the bunch. After removing these particles, corresponding to $6 \%$ of the total charge, the remainder of the beam experiences a projected emittance growth of $0.04 \%$, which is near the numerical resolution of the simulation.

Next, we simulate the same system after including nonlinear magnetic optics through 5th order. In addition, we include transient CSR effects due to bend entry and exit transitions by using the wakefield model described in [7]. The inverse problem (76) is solved for the optimized initial longitudinal density, and we compensate for nonlinear effects by using the initial energy profile given by (45). For comparison, we also use a uniform (flattop) bunch with the same end-to-end bunch length as the optimized profile, and a Gaussian bunch with the same rms bunch length as the optimized profile. The three longitudinal profiles are shown together in Fig. 10.
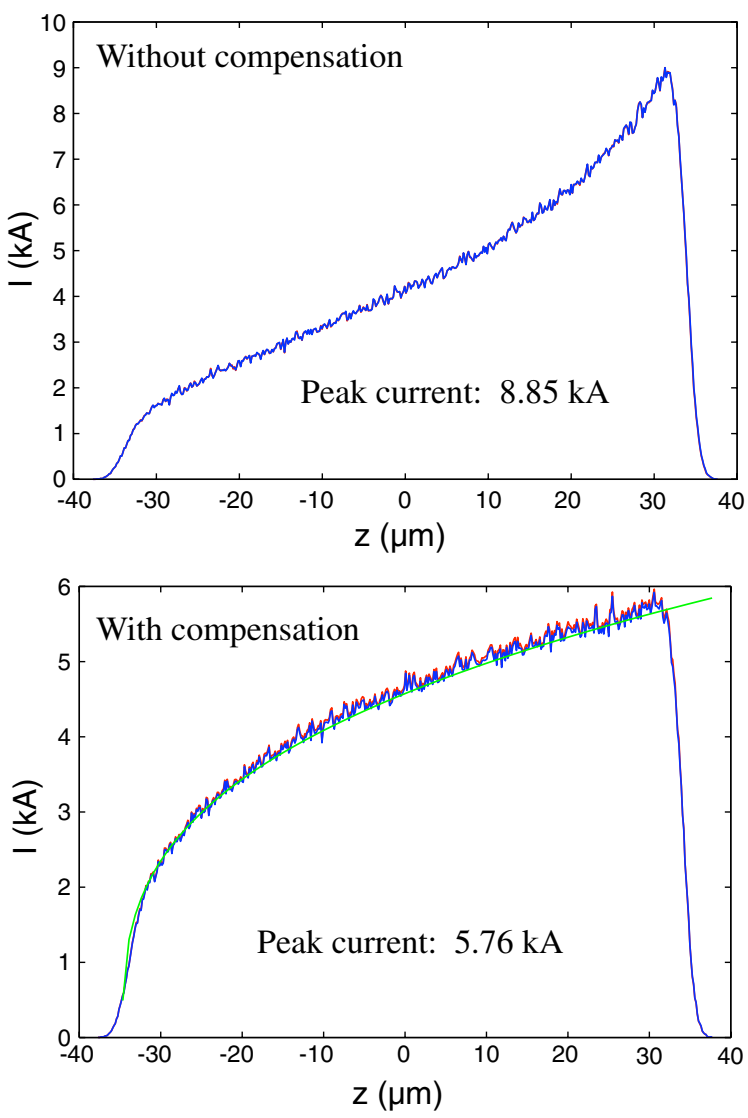

FIG. 9. The current profile at the entry to Bend 4 (blue) is shown both without (upper) and with (lower) compensation for the lattice nonlinear optics, as described in Sec. III B. The lower result is shown together with the compressed optimal current profile (green) of (15), indicating that the initial density profile is preserved.

We obtain for the Gaussian beam a final projected horizontal emittance growth of $55 \%$, for the uniform beam $8 \%$, and for the optimized beam $0.9 \%$. The emittance growth of the Gaussian beam is comparable to the benchmark results for ELEGANT described in [22]. This emittance

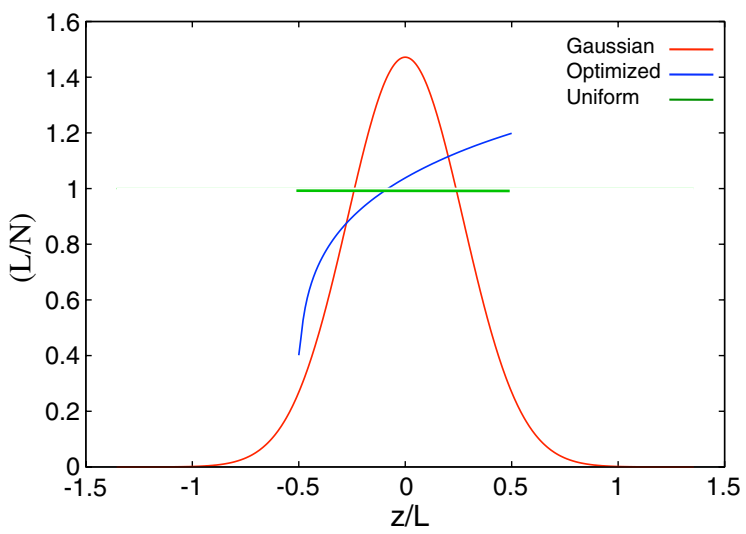

FIG. 10. Three initial current profiles used with the parameters shown in Table I for simulation in the chicane of Fig. 6. 
growth is due to the transverse misalignment of longitudinal slice centroids, as apparent in Fig. 11, which illustrates the transverse-longitudinal correlations in the three beam distributions at the exit of the system. The blue lines indicate the predicted centroid offsets given by (67), the green points denote particles in the final beam distribution, and the red points denote particles in the beam core that are used to calculate the final projected emittance. A detailed analysis of the projected emittance growth using the model of the previous section is provided in Appendix C.
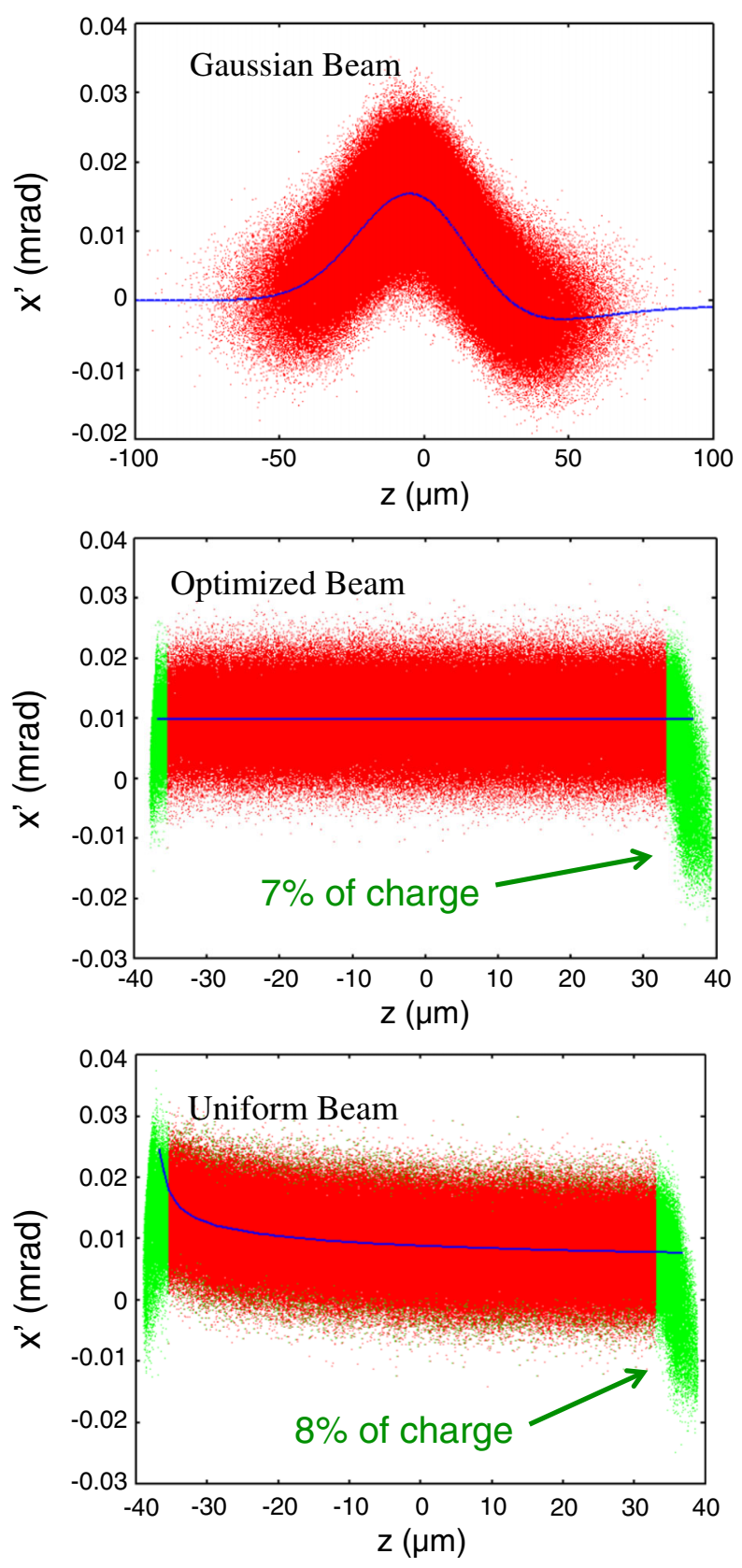

FIG. 11. Projection of the final beam distribution at the exit of the system shown in Fig. 6 into the $x^{\prime}-z$ phase plane for the three initial profiles shown in Fig. 10.
Thus, the bunch with an optimized longitudinal density profile experiences a projected emittance growth that is smaller by more than an order of magnitude relative to bunches with comparable Gaussian and uniform profiles, and it is clear that this is due to careful control of the longitudinal slice dynamics.

\section{DISCUSSION AND SUMMARY}

We have shown that careful preparation of the initial beam current profile can be used to generate a bunch whose core experiences suppressed transverse emittance growth in the presence of longitudinal CSR within a chicane $(<1 \%)$. For the limiting case of an ultrarelativistic beam undergoing ideal compression in the presence of steadystate CSR, this emittance growth can be eliminated. For the case of nonideal compression, the presence of initial energy spread and transverse emittance will lead to longitudinal spreading of the beam profile, resulting in emittance growth that is confined to particles located in small regions at the head and tail of the bunch. It is speculated that the desired longitudinal distribution (15) might be prepared using longitudinal laser shaping or by using a longitudinaltransverse emittance exchanger, although the mechanism for preparing such an initial current pulse deserves further exploration.

The results of this paper make use of a 1D model for the longitudinal CSR wakefield, which is known to be valid under moderate compression when $\sigma_{x} \ll \sigma_{z}\left(R / \sigma_{z}\right)^{1 / 3}$, where $\sigma_{x}$ and $\sigma_{z}$ are the instantaneous horizontal and longitudinal rms beam sizes [24]. In the final bend of the Berlin-Zeuthen chicane, where CSR effects are largest, this condition is easily satisfied. A free-space model of the CSR interaction is also used, so that the shielding effect of the vacuum chamber walls has been neglected. This effect becomes significant when the vacuum chamber gap height is comparable to or less than $\left(R \sigma_{z}^{2}\right)^{1 / 3}$ [15]. In this case, the formalism of Sec. IV can be used after modifying the function $K_{\mathrm{CSR}}$ to include the contribution of image charges present on the chamber walls $[6,10]$.

In an FEL beam delivery system, a beam with the longitudinal density (15) will generate wakefields due to other effects in various sections of the lattice, and the discontinuous current profile at the head of the bunch might be a cause for concern. We note that the procedure described in Sec. IV can, in principle, be generalized to include the effects of these other wakefields. In the future, this topic deserves to be studied in conjunction with numerical simulation of an optimized beam through a complete FEL system.

Finally, we remark that the procedure described here can also be used to prepare an initial current pulse that will generate a steady-state CSR wakefield that varies linearly across the length of the bunch. The resulting wakefieldinduced energy chirp will result in controlled linear $x-z$ and $x^{\prime}-z$ correlations in the beam distribution, which 
could in principle be removed using standard linear optics [13]. The advantage of this procedure is that one has an additional degree of freedom corresponding to the value of the wakefield-induced energy chirp, which can be tuned to allow for an initial current pulse with more desirable features, such as continuity. A treatment of this approach in the ultrarelativistic limit is described in Appendix D.

\section{ACKNOWLEDGMENTS}

This work was supported by U.S. Department of Energy Grant No. DE-FG02-96ER40949.

\section{APPENDIX A: INTEGRAL EQUATIONS}

A Volterra integral equation of the first kind for the function $\phi$ on an interval $[a, b]$ takes the form

$$
\int_{a}^{z} \phi\left(z^{\prime}\right) K\left(z, z^{\prime}\right) d z^{\prime}=f(z)
$$

where $z \in[a, b]$ and the integral kernel $K$ is a known function of two variables. Such equations are classified according to the behavior of the function $K$.

As a special case, the generalized Abel integral equation $[16,25]$ for a function $\phi$ on an interval $[a, b]$ takes the form

$$
\int_{a}^{z} \frac{\phi\left(z^{\prime}\right)}{\left(z-z^{\prime}\right)^{\mu}} d z^{\prime}=f(z), \quad 0<\mu<1,
$$

where $z \in[a, b]$ and $f$ is absolutely continuous on $(a, b)$. It can be shown that there exists a unique solution $\phi \in L^{1}(a, b)$ given by [16]

$$
\phi(z)=\frac{\sin \mu \pi}{\pi}\left[\frac{f(a)}{(z-a)^{1-\mu}}+\int_{a}^{z} \frac{f^{\prime}(t) d t}{(z-t)^{1-\mu}}\right] .
$$

This result is used in Sec. II A to determine the unique analytical solution for the longitudinal density profile that generates a uniform ultrarelativistic CSR wakefield.

Integral equations of the form (A1) can lead to ill-posed problems that are difficult to invert numerically; we refer the reader to the literature on regularization for a proper treatment of these difficulties. In Sec. II B, numerical convergence is problematic since the function $f$ is taken to be a constant and $K$ is continuous. Under these conditions, we note that an exact solution exists only if $\lim _{z \rightarrow a} f(z)=0$. This issue can be avoided by replacing (A1) by the regularized problem:

$$
\int_{a}^{z} \phi\left(z^{\prime}\right) K\left(z, z^{\prime}\right) d z^{\prime}=f(z)-f(a) \frac{K(z, a)}{K(a, a)},
$$

provided $K(a, a) \neq 0$. For the kernel $K_{\mathrm{CSR}}$ described in this paper, the problem (A4) is numerically well behaved in the continuum limit, and gives a solution that is indistinguishable from the approximate solution of (A1).
APPENDIX B: PROJECTED EMITTANCE MODEL

The projected horizontal, unnormalized rms emittance $\epsilon$ is defined by

$$
\epsilon^{2}=\left\langle(x-\bar{x})^{2}\right\rangle\left\langle\left(x^{\prime}-\bar{x}^{\prime}\right)^{2}\right\rangle-\left\langle(x-\bar{x})\left(x^{\prime}-\bar{x}^{\prime}\right)\right\rangle^{2},
$$

where $\bar{x}$ and $\bar{x}^{\prime}$ are the coordinates of the bunch centroid in the transverse phase space:

$$
\bar{x}=\langle x\rangle, \quad \bar{x}^{\prime}=\left\langle x^{\prime}\right\rangle,
$$

and averages denoted $\langle\cdot\rangle$ are taken with respect to the distribution function of the entire beam. Similarly, the slice emittance corresponding to a slice with longitudinal coordinate $z_{j}$ is given by

$$
\epsilon_{j}^{2}=\left\langle\left(x-x_{j}\right)^{2}\right\rangle_{j}\left\langle\left(x^{\prime}-x_{j}^{\prime}\right)^{2}\right\rangle_{j}-\left\langle\left(x-x_{j}\right)\left(x^{\prime}-x_{j}^{\prime}\right)\right\rangle_{j}^{2},
$$

where $x_{j}$ and $x_{j}^{\prime}$ are the coordinates of the centroid of the slice in the transverse phase space:

$$
x_{j}=\langle x\rangle_{j}, \quad x_{j}^{\prime}=\left\langle x^{\prime}\right\rangle_{j},
$$

and we have used the notation $\langle\cdot\rangle_{j}=\langle\cdot\rangle_{z_{j}}$ to the denote that an average is taken over the slice corresponding to the longitudinal coordinate $z_{j}$.

Suppose that the slice moments are identical for each slice, except for possible translations of the slice centroids. We wish to determine the relationship between the slice emittance $\epsilon_{0}=\epsilon_{j}$ (which is identical for each slice) and the projected emittance $\epsilon$. We have the following expressions for the second-order moments of the beam:

$$
\begin{aligned}
\left\langle(x-\bar{x})^{2}\right\rangle & =\frac{1}{N} \sum_{j} \lambda_{j}\left\langle(x-\bar{x})^{2}\right\rangle_{j} \\
& =\frac{1}{N} \sum_{j} \lambda_{j}\left\langle\left[\left(x-x_{j}\right)+\left(x_{j}-\bar{x}\right)\right]^{2}\right\rangle_{j} \\
& =\sigma_{x \perp}^{2}+\sigma_{x \|}^{2},
\end{aligned}
$$

where

$$
\sigma_{x \perp}^{2}=\left\langle\left(x-x_{j}\right)^{2}\right\rangle_{j}, \quad \sigma_{x \|}^{2}=\frac{1}{N} \sum_{j=1}^{N} \lambda_{j}\left(x_{j}-\bar{x}\right)^{2} .
$$

Similarly,

$$
\left\langle\left(x^{\prime}-\bar{x}^{\prime}\right)^{2}\right\rangle=\sigma_{x^{\prime} \perp}^{2}+\sigma_{x^{\prime} \|}^{2},
$$

where

$$
\sigma_{x^{\prime} \perp}^{2}=\left\langle\left(x^{\prime}-x_{j}^{\prime}\right)^{2}\right\rangle_{j}, \quad \sigma_{x^{\prime} \|}^{2}=\frac{1}{N} \sum_{j=1}^{N} \lambda_{j}\left(x_{j}^{\prime}-\bar{x}^{\prime}\right)^{2} .
$$

Also, 


$$
\begin{aligned}
& \left\langle(x-\bar{x})\left(x^{\prime}-\bar{x}^{\prime}\right)\right\rangle=\frac{1}{N} \sum_{j} \lambda_{j}\left\langle(x-\bar{x})\left(x^{\prime}-\bar{x}^{\prime}\right)\right\rangle_{j} \\
= & \frac{1}{N} \sum_{j} \lambda_{j}\left\langle\left[\left(x-x_{j}\right)+\left(x_{j}-\bar{x}\right)\right]\left[\left(x^{\prime}-x_{j}^{\prime}\right)+\left(x_{j}^{\prime}-\bar{x}^{\prime}\right)\right]\right\rangle_{j} \\
= & \left\langle X X^{\prime}\right\rangle_{\perp}+\left\langle X X^{\prime}\right\rangle_{\|},
\end{aligned}
$$

where

$$
\begin{array}{r}
\left\langle X X^{\prime}\right\rangle_{\perp}=\left\langle\left(x-x_{j}\right)\left(x^{\prime}-x_{j}^{\prime}\right)\right\rangle_{j}, \\
\left\langle X X^{\prime}\right\rangle_{\|}=\frac{1}{N} \sum_{j} \lambda_{j}\left(x_{j}-\bar{x}\right)\left(x_{j}^{\prime}-\bar{x}^{\prime}\right) .
\end{array}
$$

This gives then

$$
\begin{aligned}
\epsilon^{2} & =\left(\sigma_{x \perp}^{2}+\sigma_{x \|}^{2}\right)\left(\sigma_{x^{\prime} \perp}^{2}+\sigma_{x^{\prime} \|}^{2}\right)-\left(\left\langle X X^{\prime}\right\rangle_{\perp}+\left\langle X X^{\prime}\right\rangle_{\|}\right)^{2} \\
& =\epsilon_{0}^{2}+\sigma_{x \|}^{2} \sigma_{x^{\prime} \perp}^{2}+\sigma_{x \perp}^{2} \sigma_{x^{\prime} \|}^{2}-2\left\langle X X^{\prime}\right\rangle_{\perp}\left\langle X X^{\prime}\right\rangle_{\|}+\epsilon_{\|}^{2},
\end{aligned}
$$

where

$$
\epsilon_{0}^{2}=\sigma_{x \perp}^{2} \sigma_{x^{\prime} \perp}^{2}-\left\langle X X^{\prime}\right\rangle_{\perp}^{2}, \quad \epsilon_{\|}^{2}=\sigma_{x \|}^{2} \sigma_{x^{\prime} \|}^{2}-\left\langle X X^{\prime}\right\rangle_{\|}^{2} .
$$

Defining the slice Twiss functions by

$$
\sigma_{x \perp}^{2}=\beta \epsilon_{0}, \quad \sigma_{x^{\prime} \perp}^{2}=\gamma \epsilon_{0}, \quad\left\langle X X^{\prime}\right\rangle_{\perp}=-\alpha \epsilon_{0}
$$

gives the following result for the projected emittance of the beam:

$$
\epsilon^{2}=\epsilon_{0}^{2}+\epsilon_{0}\left(\beta \sigma_{x^{\prime} \|}^{2}+\gamma \sigma_{x \|}^{2}+2 \alpha\left\langle X X^{\prime}\right\rangle_{\|}\right)+\epsilon_{\|}^{2} .
$$

\section{APPENDIX C: PROJECTED EMITTANCE ESTIMATES}

In this Appendix, we provide emittance estimates for the Berlin-Zeuthen benchmark chicane for several longitudinal density profiles using the slice-evolution model of emittance growth described in the main text.

\section{Density profiles}

The following longitudinal density profiles are normalized according to (1), where $N$ denotes the number of particles within a bunch. Here $\sigma_{z}$ denotes the rms bunch length and $L$ denotes the end-to-end (total) bunch length.

Gaussian:

$$
\lambda(z)=\frac{N}{\sigma_{z} \sqrt{2 \pi}} \exp \left(-z^{2} / 2 \sigma_{z}^{2}\right)
$$

Uniform:

$$
\lambda(z)=\left\{\begin{array}{ll}
N / L & |z| \leq L / 2 \\
0 & |z|>L / 2,
\end{array} \quad \sigma_{z}=L / \sqrt{12}\right.
$$

Parabolic:

$$
\lambda(z)=\left\{\begin{array}{ll}
\frac{3 N}{2 L}\left[1-\left(\frac{2 z}{L}\right)^{2}\right] & |z| \leq L / 2 \\
0 & |z|>L / 2,
\end{array} \quad \sigma_{z}=L / \sqrt{20} .\right.
$$

Optimal:

$$
\lambda(z)=\left\{\begin{array}{ll}
\frac{4 N}{3 L}\left(\frac{z}{L}+\frac{1}{2}\right)^{1 / 3} & |z| \leq L / 2 \\
0 & |z|>L / 2,
\end{array} \sigma_{z}=\frac{3}{7} \sqrt{\frac{2}{5}} L .\right.
$$

\section{Steady-state wakefield $(\gamma \rightarrow \infty)$}

In this section, we give the steady-state CSR wakefield (8) generated by bunches with the density profiles $(\mathrm{C} 1)-(\mathrm{C} 4)$. Following the notation in the main text, we define

$$
\kappa=\frac{2 r_{c} m c^{2}}{3^{1 / 3} R^{2 / 3}},
$$

where $r_{c}$ is the classical electron radius, $R$ is the bending radius, and $m c^{2}$ is the electron rest energy. The wakefield for each case is given below.

Gaussian:

$$
W(z)=\frac{N \kappa}{\sigma_{z}^{4 / 3}} \tilde{W}\left(z / \sigma_{z}\right)
$$

where

$$
\begin{aligned}
\tilde{W}(z)= & \frac{1}{4 \sqrt{\pi}}\left[-2^{4 / 3} \Gamma\left(\frac{5}{6}\right){ }_{1} F_{1}\left(\frac{2}{3}, \frac{1}{2},-\frac{z^{2}}{2}\right)\right. \\
& \left.+2^{5 / 6} z \Gamma\left(\frac{4}{3}\right){ }_{1} F_{1}\left(\frac{7}{6}, \frac{3}{2},-\frac{z^{2}}{2}\right)\right] .
\end{aligned}
$$

Uniform:

$$
W(z)=-\frac{N \kappa}{L^{4 / 3}}\left(\frac{z}{L}+\frac{1}{2}\right)^{-1 / 3}, \quad|z| \leq L / 2 .
$$

Parabolic:

$$
W(z)=\frac{N \kappa}{L^{4 / 3}} \frac{18}{5}\left(\frac{z}{L}+\frac{1}{2}\right)^{2 / 3}\left(3 \frac{z}{L}-1\right), \quad|z| \leq L / 2 .
$$

Optimal:

$$
W(z)=-\frac{8}{9 \sqrt{3}} \frac{N \pi \kappa}{L^{4 / 3}}, \quad|z| \leq L / 2 .
$$

\section{CSR-induced energy change and rms spread}

In this section, we evaluate the quantities:

$$
\langle W\rangle_{\|}, \quad \sigma_{W}=\sqrt{\left\langle W^{2}\right\rangle_{\|}-\langle W\rangle_{\|}^{2}},
$$

for each longitudinal density (C1)-(C4). We will compare: (i) four bunches with the density profiles $(\mathrm{C} 1)-(\mathrm{C} 4)$ and 
TABLE II. Comparison of CSR-induced energy change and rms CSR-induced energy spread for densities with matched rms bunch length.

\begin{tabular}{lcc}
\hline \hline Density & $\left\langle W / W_{0}\right\rangle_{\|}$ & $\sigma_{W} / W_{0}$ \\
\hline Gaussian & -0.893627 & 0.627212 \\
Uniform & -1.011881 & 0.584210 \\
Parabolic & -0.832947 & 0.450983 \\
Optimal & -1.000000 & 0.000000 \\
\hline \hline
\end{tabular}

TABLE III. Comparison of CSR-induced energy change and rms CSR-induced energy spread for densities with matched total bunch length.

\begin{tabular}{lcc}
\hline \hline Density & $\left\langle W / W_{0}\right\rangle_{\|}$ & $\sigma_{W} / W_{0}$ \\
\hline Uniform & -0.930368 & 0.537148 \\
Parabolic & -1.076568 & 0.582887 \\
Optimal & -1.000000 & 0.000000 \\
\hline \hline
\end{tabular}

matched rms bunch length; and (ii) three bunches with the density profiles $(\mathrm{C} 2)-(\mathrm{C} 4)$ and matched end-to-end bunch length.

The number of particles is identical in each case, and we normalize by the magnitude of the steady-state wakefield that is generated by the optimal density:

$$
W_{0}=\frac{8}{9 \sqrt{3}} \frac{N \pi \kappa}{L^{4 / 3}},
$$

where $L$ is the total length of the optimal bunch. The results are shown in Tables II and III. Notice that the rms variation in the CSR-induced energy change per unit length is zero for the optimal density profile but comparable for the other density profiles considered here.

\section{CSR-induced emittance growth}

The final emittance is given by

$$
\epsilon^{2}=\epsilon_{0}^{2}+\epsilon_{0}\left(\beta \sigma_{x^{\prime} \|}^{2}+\gamma \sigma_{x \|}^{2}+2 \alpha\left\langle X X^{\prime}\right\rangle_{\|}\right)+\epsilon_{\| \cdot}^{2}
$$

To use this model, we must numerically compute the quantities $\sigma_{x \|}, \sigma_{x^{\prime} \|}$, and $\left\langle X X^{\prime}\right\rangle_{\|}$, and we need the Twiss functions at the exit of the system (the end of the final drift). Direct evaluation using the linear transfer matrix for the Berlin-Zeuthen chicane gives the Twiss functions:

$$
\alpha=-0.315, \quad \beta=5.67 \mathrm{~m}, \quad \gamma=0.194 \mathrm{~m}^{-1} \text {. }
$$

We evaluate the effect of the slice centroid offsets by evaluating the quantities:

$$
\sigma_{x \|}, \quad \sigma_{x^{\prime} \|}, \quad r_{\|}=\left\langle X X^{\prime}\right\rangle_{\|}, \quad \epsilon_{\|} .
$$

The final CSR-induced offset of a longitudinal slice with initial longitudinal coordinate $u$ is given by

$$
\begin{aligned}
\Delta x^{\prime}(u) & =\frac{1}{E} \int_{-\infty}^{u} \lambda_{0}\left(u^{\prime}\right) K_{\mathrm{CSR}}^{\mathrm{int}, x^{\prime}}\left(u, u^{\prime}\right) d u^{\prime}, \\
\Delta x(u) & =\frac{1}{E} \int_{-\infty}^{u} \lambda_{0}\left(u^{\prime}\right) K_{\mathrm{CSR}}^{\mathrm{int}, x}\left(u, u^{\prime}\right) d u^{\prime},
\end{aligned}
$$

where

$$
\begin{aligned}
K_{\mathrm{CSR}}^{\mathrm{int}, x^{\prime}}\left(u, u^{\prime}\right)= & \int d \tau R_{26}\left(\tau \rightarrow s_{f}\right) K_{\mathrm{CSR}}[u / C(\tau) \\
& \left.+\tau, u^{\prime} / C(\tau)+\tau\right], \\
K_{\mathrm{CSR}}^{\mathrm{int}, x}\left(u, u^{\prime}\right)= & \int d \tau R_{16}\left(\tau \rightarrow s_{f}\right) K_{\mathrm{CSR}}[u / C(\tau) \\
& \left.+\tau, u^{\prime} / C(\tau)+\tau\right],
\end{aligned}
$$

and the integrals are taken over the length of the lattice. These integrals can be computed numerically and used to evaluate (C15), and the results are shown in Tables IV and V.

In Table IV, each density profile is given the same initial rms bunch length. The emittance growth of $57 \%$ that is predicted for the Gaussian bunch is in agreement with the IMPACT-Z simulation result of 55\% described in Sec. V.

In Table V, each density profile is given the same initial total bunch length. When expressed in terms of the final (compressed) longitudinal coordinate $z / C$, each density has been truncated outside a range $\left[z_{\min }, z_{\max }\right]$ for the purpose of emittance calculation, where the values $z_{\min }$ and $z_{\max }$ are chosen in order to correspond to the numerical procedure used to compute emittance from the particle distributions resulting from IMPACT-Z. (See Fig. 11.) Simulation using IMPACT-Z gives a final projected emittance growth of $<0.9 \%$ for the optimized bunch and $8 \%$ for a uniform bunch with matched total bunch length. It follows that for these parameters, the slice model of emittance

TABLE IV. Relative CSR-induced emittance growth at the end of the final drift of the Berlin-Zeuthen benchmark chicane, assuming a matched initial $\mathrm{rms}$ bunch length of $200 \mu \mathrm{m}$.

\begin{tabular}{lccccc}
\hline \hline Density & $\sigma_{x \|}(\mu \mathrm{m})$ & $\sigma_{x^{\prime} \|}(\mu \mathrm{r})$ & $r_{\|}(\mu \mathrm{m}-\mu \mathrm{r})$ & $\epsilon_{\|}(\mu \mathrm{m}-\mu \mathrm{r})$ & $\Delta \epsilon / \epsilon_{0}$ \\
\hline Gaussian & 2.164 & 5.241 & 9.166 & 6.682 & 0.575 \\
Uniform & 1.972 & 2.938 & 5.507 & 1.803 & 0.205 \\
Parabolic & 1.824 & 3.571 & 5.141 & 4.001 & 0.298 \\
Optimal & 1.120 & 0.000 & 0.000 & 0.000 & 0.001 \\
\hline \hline
\end{tabular}

TABLE V. Relative CSR-induced emittance growth at the end of the final drift of the Berlin-Zeuthen benchmark chicane, assuming a matched initial end-to-end bunch length of $0.738 \mathrm{~mm}$ (after truncating slices near the head and tail as seen in Fig. 11).

\begin{tabular}{lccccc}
\hline \hline Density & $\sigma_{x \|}(\mu \mathrm{m})$ & $\sigma_{x^{\prime} \|}(\mu \mathrm{r})$ & $r_{\|}(\mu \mathrm{m}-\mu \mathrm{r})$ & $\epsilon_{\|}(\mu \mathrm{m}-\mu \mathrm{r})$ & $\Delta \epsilon / \epsilon_{0}$ \\
\hline Uniform & 1.597 & 1.882 & 2.971 & 0.5899 & 0.088 \\
Parabolic & 2.290 & 4.431 & 8.401 & 5.690 & 0.432 \\
Optimal & 1.092 & 0.000 & 0.000 & 0.000 & 0.001 \\
\hline \hline
\end{tabular}


growth gives a prediction of the final projected emittance in the beam core that is accurate to within $1 \%$.

\section{The steady-state ultrarelativistic limit}

In the steady-state ultrarelativistic limit, the integrals appearing in (C17) take a simpler form. Since transient CSR within the drifts is neglected, the integrands vanish for values of $\tau$ corresponding to locations within a drift. For values of $\tau$ corresponding to locations within a bend of radius $R$,

$$
K_{\mathrm{CSR}}\left[u / C(\tau)+\tau, u^{\prime} / C(\tau)+\tau\right]=C(\tau)^{4 / 3} K_{\mathrm{CSR}}^{\circ}\left(u-u^{\prime}\right),
$$

where $K_{\mathrm{CSR}}^{\circ}$ is given by (9). Assuming that the four bends of the chicane have the same radius of curvature, this gives

$$
\begin{aligned}
& K_{\mathrm{CSR}}^{\mathrm{int}, x^{\prime}}\left(u, u^{\prime}\right)=K_{\mathrm{CSR}}^{\circ}\left(u-u^{\prime}\right) \Sigma^{x^{\prime}}, \\
& K_{\mathrm{CSR}}^{\mathrm{int}, x}\left(u, u^{\prime}\right)=K_{\mathrm{CSR}}^{\circ}\left(u-u^{\prime}\right) \Sigma^{x},
\end{aligned}
$$

where

$$
\begin{aligned}
\Sigma^{x^{\prime}} & =\sum_{j=1}^{4} \int_{B_{j}} R_{26}(\tau \rightarrow s) C(\tau)^{4 / 3} d \tau, \\
\Sigma^{x} & =\sum_{j=1}^{4} \int_{B_{j}} R_{16}(\tau \rightarrow s) C(\tau)^{4 / 3} d \tau,
\end{aligned}
$$

and the notation $B_{j}, j=1, \ldots, 4$ denotes that the integral is taken over the length of bend $j$. Now let

$$
W_{1}(u)=\frac{1}{E} \int_{-\infty}^{u} \lambda_{0}\left(u^{\prime}\right) K_{\mathrm{CSR}}^{\circ}\left(u-u^{\prime}\right) d u^{\prime}
$$

denote the steady-state CSR wakefield generated by the initial density $\lambda_{0}$ within Bend 1 of the chicane (after scaling by the beam energy). Then it follows from (C16) that

$$
\Delta x^{\prime}(u)=W_{1}(u) \Sigma^{x^{\prime}}, \quad \Delta x(u)=W_{1}(u) \Sigma^{x} .
$$

It follows that the final projected emittance in the steadystate, ultrarelativistic approximation is given by

$$
\epsilon^{2}=\epsilon_{0}^{2}+\epsilon_{0} \sigma_{W_{1}}^{2}\left(\beta \Sigma^{x^{\prime} 2}+\gamma \Sigma^{x 2}+2 \alpha \Sigma^{x} \Sigma^{x^{\prime}}\right) .
$$

The dependence of the emittance on the initial longitudinal density is determined by the quantity $\sigma_{W_{1}}$, which can be determined from Tables II and III.

In order to demonstrate the relative contribution of the CSR in each bend to the total emittance growth in the chicane, Table VI gives the contribution made by each bend to the integrals $\Sigma^{x}$ and $\Sigma^{x^{\prime}}$ appearing in (C19). Using the final line of Table VI in (C22) to calculate the relative emittance growth for a Gaussian bunch, we find $\Delta \epsilon / \epsilon=0.185$, which is consistent with the steady-state, ultrarelativistic simulation result of $19 \%$. Notice that the dominant term comes from the contribution made to $\Sigma^{x^{\prime}}$
TABLE VI. Contributions to the quantities (C19) that determine the relative CSR-induced emittance growth due to each of the four bends of the Berlin-Zeuthen benchmark chicane.

\begin{tabular}{lrr}
\hline \hline Bend & $\Sigma^{x^{\prime}}(\mathrm{m})$ & $\Sigma^{x}\left(\mathrm{~m}^{2}\right)$ \\
\hline B1 & -0.0120 & -0.1787 \\
B2 & -0.0152 & -0.3336 \\
B3 & 0.1065 & -0.0249 \\
B4 & 0.2615 & 0.5670 \\
Total & 0.3407 & 0.0297 \\
\hline \hline
\end{tabular}

by Bend 4 . However, using this contribution alone (with $\left.\Sigma^{x}=0\right)$ gives the underestimate $\Delta \epsilon / \epsilon=0.114$.

Let us relate $(\mathrm{C} 22)$ to the approximate expression for the emittance growth generated within a single bend, as given in (5). Suppose that the first term in parentheses in (C22) dominates. This gives

$$
\epsilon^{2} \approx \epsilon_{0}^{2}+\epsilon_{0} \sigma_{W_{1}}^{2} \beta \Sigma^{x^{\prime} 2} .
$$

Let us now assume that the effect of CSR is dominated by the dynamics in the fourth bend of the chicane. That is,

$$
\Sigma^{x^{\prime}} \approx \int_{B_{4}} R_{26}(\tau \rightarrow s) C(\tau)^{4 / 3} d \tau .
$$

Since the compression factor is nearly unchanged within the final bend, we integrate over the matrix element $R_{26}(\tau \rightarrow s)=\sin [(s-\tau) / R]$ to obtain

$$
\Sigma^{x^{\prime}} \approx C^{4 / 3} R(1-\cos \theta),
$$

where $\theta$ is the bend angle and $C$ is the compression factor at the chicane exit. Making the usual small-angle approximation in $\theta$ and using this result in (C23) gives

$$
\epsilon^{2}=\epsilon_{0}^{2}+\epsilon_{0} \sigma_{W_{1}}^{2} \frac{C^{8 / 3} L_{b}^{3} \theta^{2}}{4}\left(\frac{\beta}{L_{b}}\right) .
$$

However, under the model of ideal compression (36) that is assumed here, the wakefield at a lattice location $s$ is related to the wakefield within the first bend (C20) by

$$
W(z, s)=C(s)^{4 / 3} W_{1}[z C(s)] E,
$$

so that the CSR-induced energy change per unit length at the exit of the final bend is given by

$$
\sigma_{W}=C^{4 / 3} \sigma_{W_{1}} E .
$$

Using (C27) in (C25) gives finally

$$
\epsilon \approx \sqrt{\epsilon_{0}^{2}+\epsilon_{0} \beta\left(\theta L_{b} \sigma_{W} / 2 E\right)^{2}} .
$$

This is to be compared with (5). Noting that the CSRinduced energy spread at the exit of the bend is given by $\sigma_{E}=L_{b} \sigma_{W}$, we see that these expressions apparently differ due to the factor of 2 appearing in the denominator of (C28). The disagreement occurs because the estimate given in (5) implicitly assumes that the beam experiences 
an instantaneous CSR-induced energy spread at the bend entrance. In reality, the CSR-induced energy spread accumulates linearly over the length of the bend; the two expressions agree provided that one uses the average of the beam CSR-induced energy spread along the bend length (given by $\sigma_{E}=L_{b} \sigma_{W} / 2$ ) in evaluating (5).

Computing the emittance growth using this expression gives for a Gaussian bunch $\Delta \epsilon / \epsilon \approx 0.104$. It follows that to obtain an accurate estimate (within $50 \%$ ) of the CSR-induced emittance growth in the chicane requires that one consider the effects of CSR within Bends 3 and 4, as well as the transient CSR within the drifts.

\section{APPENDIX D: GENERATING A LINEAR ENERGY CHIRP}

In some cases, the bunch may possess a linear energybunch length correlation that one wishes to remove. We will see that for an appropriate choice of current profile, this energy chirp may be removed by exploiting the longitudinal CSR wakefield [26]. Alternatively, we may use the CSR wakefield to add a desired energy chirp to the beam. In either case, we wish to obtain a longitudinal wakefield of the form

$$
W(z)=h z-W_{0}, \quad z \in[a, b],
$$

where $h=d W / d z$ is the desired energy chirp per unit length, and $W_{0}$ is a net wakefield offset. Making use of the ultrarelativistic expression (8) for the steady-state CSR wakefield, a solution is obtained for the longitudinal density $\lambda$ by using the result of Appendix A in terms of the dimensionless parameter:

$$
\hat{h}=\frac{27 h L^{7 / 3}}{112 \sqrt{3} N \pi \kappa},
$$

where $L=b-a$ is the total length of the bunch and $\kappa$ is given by (11). Let us choose our coordinate system so that $a=-L / 2$ and $b=L / 2$. Defining the dimensionless quantities $\bar{z}=z / L$ and $\bar{\lambda}=(L / N) \lambda$, the unique solution can be written:

$$
\bar{\lambda}(\bar{z})=\left(\bar{z}+\frac{1}{2}\right)^{1 / 3}\left[\frac{4}{3}-\hat{h}(14 \bar{z}-1)\right], \quad \bar{z} \in[-1 / 2,1 / 2] .
$$

This solution possesses two zeros $\bar{z}_{1}$ and $\bar{z}_{2}$ at $\bar{z}_{1}=-1 / 2$ (the bunch tail) and

$$
\bar{z}_{2}=\frac{1}{14}\left(1+\frac{4}{3 \hat{h}}\right)
$$

However, a physical bunch of single-species charge carriers can produce a longitudinal density $\bar{\lambda}$ only if $\bar{\lambda} \geq 0$ everywhere. Enforcing this requirement therefore limits the energy chirp values $\hat{h}$ that can be attained to the range:

$$
-\frac{1}{6} \leq \hat{h} \leq \frac{2}{9} \text {. }
$$

The wakefield offset associated with a given energy chirp $\hat{h}$ is given by

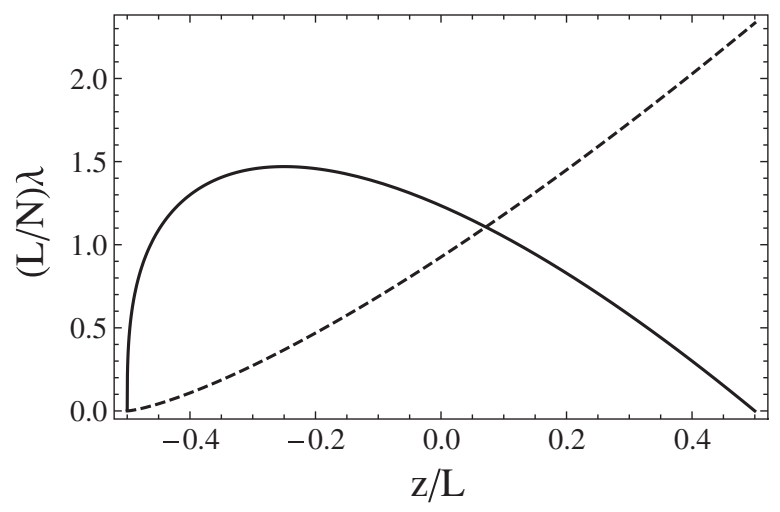

FIG. 12. Solution (D3) for $\hat{h}=2 / 9$ (solid) and $\hat{h}=-1 / 6$ (dashed).

$$
W_{0}=\frac{8}{9 \sqrt{3}} \frac{N \pi \kappa}{L^{4 / 3}}(1-\hat{h})>0 .
$$

These results reduce to those of Sec. II in the limit $\hat{h} \rightarrow 0$.

Of particular interest are the extreme cases given by the upper and lower limits on the chirp given in (D5). The case $\hat{h}=-1 / 6$ produces a longitudinal density whose derivative is smooth at the bunch tail $(\bar{z}=-1 / 2)$, while the case $\hat{h}=2 / 9$ produces a longitudinal density which is continuous at the bunch head $(\bar{z}=1 / 2)$. These cases are shown together in Fig. 12.

[1] R. Talman, Phys. Rev. Lett. 56, 1429 (1986).

[2] P. Emma and R. Brinkmann, in Proceedings of the 1997 Particle Accelerator Conference, Vancouver, BC, Canada (IEEE, New York, 1997), p. 1679; Stanford Linear Accelerator Center Report No. SLAC-PUB-7554, 1997.

[3] H. Braun, F. Chautard, R. Corsini, T. Raubenheimer, and P. Tenenbaum, Phys. Rev. Lett. 84, 658 (2000).

[4] S. Heifets, G. Stupakov, and S. Krinsky, Phys. Rev. ST Accel. Beams 5, 064401 (2002).

[5] Z. Huang and K. J. Kim, Phys. Rev. ST Accel. Beams 5, 074401 (2002).

[6] J. Murphy, S. Krinsky, and R. Gluckstern, Part. Accel. 57, 9 (1997).

[7] E. L. Saldin, E. A. Schneidmiller, and M. V. Yurkov, Nucl. Instrum. Methods Phys. Res., Sect. A 398, 373 (1997).

[8] M. Dohlus and T. Limberg, Nucl. Instrum. Methods Phys. Res., Sect. A 393, 494 (1997).

[9] C. Mayes and G. Hoffstaetter, Phys. Rev. ST Accel. Beams 12, 024401 (2009).

[10] D. Sagan, G. Hoffstaetter, C. Mayes, and U. Sae-Ueng, Phys. Rev. ST Accel. Beams 12, 040703 (2009).

[11] V. Yakimenko, M. Fedurin, V. Litvinenko, A. Fedotov, D. Kayran, and P. Muggli, Phys. Rev. Lett. 109, 164802 (2012).

[12] D. Douglas, Thomas Jefferson National Laboratory Facility Report No. JLAB-TN-98-012, 1998. 
[13] S. Di Mitri, M. Cornacchia, and S. Spampinati, Phys. Rev. Lett. 110, 014801 (2013).

[14] M. Dohlus and T. Limberg, in Proceedings of the 2005 Particle Accelerator Conference, Knoxville, 2005 (IEEE, Piscataway, NJ, 2005), p. 1015.

[15] M. Dohlus, T. Limberg, and P. Emma, ICFA Beam Dynamics Newsletter 38, 39 (2005).

[16] A. D. Polyanin and A. V. Manzhirov, Handbook of Integral Equations (CRC Press, Boca Raton, 1998).

[17] A. Wazwaz, Linear and Nonlinear Integral Equations: Methods and Applications (Springer-Verlag, Berlin, 2011).

[18] M. Abramowitz and I. Stegun, Handbook of Mathematical Formulas and Integrals (Academic Press, New York, 1995).
[19] C. Mitchell, J. Qiang, and R. Ryne, Nucl. Instrum. Methods Phys. Res., Sect. A 715, 119 (2013).

[20] K. Flottmann et al., DESY-TESLA FEL Report No. 2001-06.

[21] W. Walter, Ordinary Differential Equations (SpringerVerlag, New York, 2002).

[22] ICFA Beam Dynamics Mini-Workshop, Berlin-Zeuthen, 2002 [http://www.desy.de/csr].

[23] J. Qiang, R. D. Ryne, S. Habib, and V. Decyk, J. Comput. Phys. 163, 434 (2000).

[24] R. Li, Phys. Rev. ST Accel. Beams 11, 024401 (2008).

[25] R. Gorenflo and S. Vessella, Abel Integral Equations: Analysis and Applications (Springer-Verlag, Berlin, 1991).

[26] R. A. Bosch, Phys. Rev. ST Accel. Beams 13, 110702 (2010). 